\title{
Transonic and supersonic ground effect aerodynamics
}

\author{
G. Doig* \\ School of Mechanical and Manufacturing Engineering, University of New South Wales, Sydney, Australia
}

\begin{abstract}
A B S T R A C T
A review of recent and historical work in the field of transonic and supersonic ground effect aerodynamics has been conducted, focussing on applied research on wings and aircraft, present and future ground transportation, projectiles, rocket sleds and other related bodies which travel in close ground proximity in the compressible regime. Methods for ground testing are described and evaluated, noting that wind tunnel testing is best performed with a symmetry model in the absence of a moving ground; sled or rail testing is ultimately preferable, though considerably more expensive. Findings are reported on shock-related ground influence on aerodynamic forces and moments in and accelerating through the transonic regime - where force reversals and the early onset of local supersonic flow is prevalent - as well as more predictable behaviours in fully supersonic to hypersonic ground effect flows.
\end{abstract}

\section{Contents}

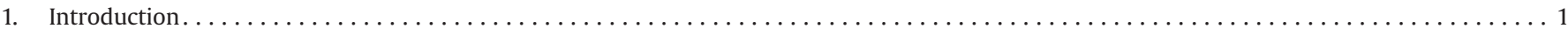

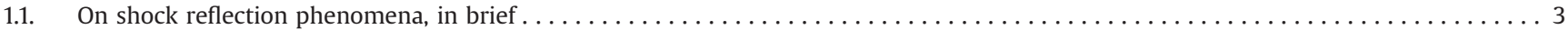

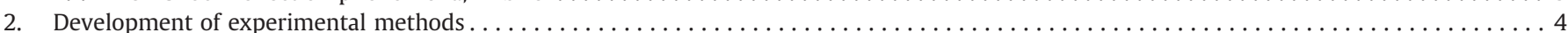

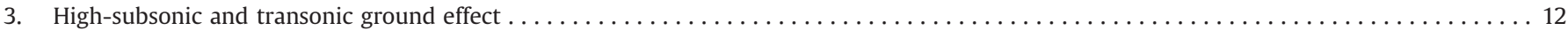

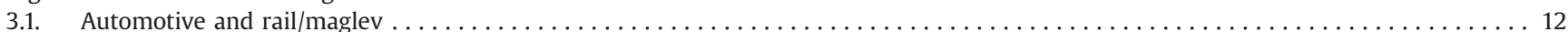

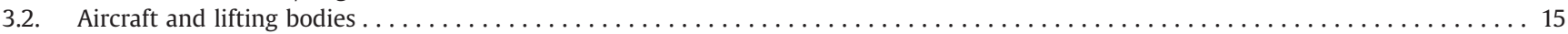

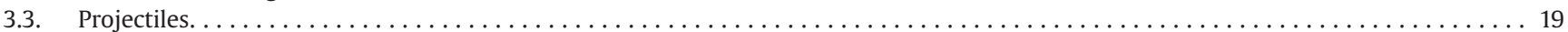

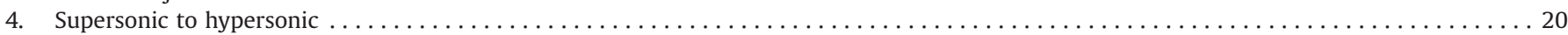

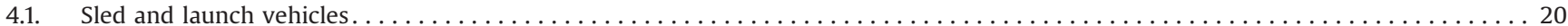

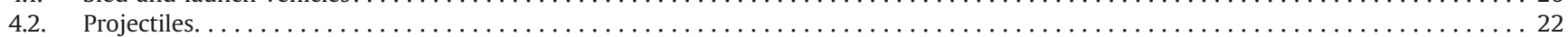

5. Conclusions ...

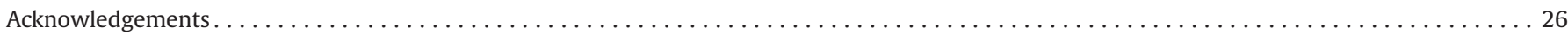

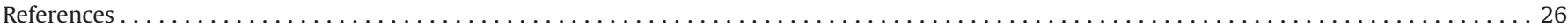

\section{Introduction}

As an object passes through a compressible fluid such as air, the aerodynamics of the body are affected by density changes in the fluid around it. These aerodynamic effects are influenced - usually exaggerated - by proximity to a ground plane, in particular when shock waves reflect from the ground to interact with the body again one or more times. Traditionally, most aeronautical ground effect research (excluding study of vertical take-off and landing

* Tel.: +6129385 5428 .

E-mail address: g.doig@unsw.edu.au
(VTOL)) has concentrated on the properties of wings in nominally incompressible flows, i.e. at relatively low subsonic Mach numbers. Applications have included aircraft in landing or takeoff modes, aircraft designed specifically to fly in ground effect, or in the case of inverted wings, high-performance racing vehicles. In these cases, proximity to the ground serves to enhance the lift (or downforce) performance of the wing, and often the overall aerodynamic efficiency as well.

Recent developments in the understanding of the aerodynamic influence of compressible ground effects and of shock/ground interaction for ground effect problems are timely, particularly given new or recurring interest in high-speed subsonic (freestream Mach number, $M_{\infty} \geq 0.4$ ) wing-in-ground effect (WIG) 


\begin{tabular}{|llll|}
\hline \multicolumn{2}{|c|}{ Nomenclature } & $f$ & frequency (Hz) \\
& & $h$ & height (m) \\
$c$ & chord (m) & $k$ & turbulent kinetic energy \\
$C_{A}$ & coefficient of axial force & $l$ & length (m) \\
$C_{D}$ & coefficient of drag & $L$ & lift force (N) \\
$C_{L}$ & coefficient of lift & $M_{\infty}$ & freestream Mach number \\
$C_{M}$ & coefficient of pitching moment & $P_{o}$ & total pressure (Pa) \\
$C_{N}$ & coefficient of force normal to the ground plane & $t$ & time (s) \\
$C_{P}$ & coefficient of pressure & $u_{\infty}$ & freestream velocity \\
$C_{Y}$ & coefficient of side force & $\alpha$ & angle of incidence \\
$C_{Z}$ & coefficient of side force (projectiles) & $\delta$ & boundary layer thickness \\
$d$ & diameter (m) & $\varepsilon$ & turbulent dissipation rate \\
$D$ & drag force (N) & $\omega$ & specific dissipation rate \\
\end{tabular}

aircraft [1], magnetic-levitation space vehicle launch systems [2], and high speed rail [3] or tube transport systems. For more esoteric applications, it has also been speculated that the shock waves from an extremely low-flying supersonic aircraft could potentially be used as a means to suppress large-scale uncontrolled fires such as forest fires [4,5], or that the use of a sonic boom from a low-flying supersonic jet could be used as a nonexplosive weapon to injure or disorient humans as part of a military operation [6].

To take aircraft as an example: in an comprehensive review of WIG aircraft aerodynamics and technology, Rozhdestvensky [1] affirms "it can be stated that little is still known with regard to GE (ground effect) at high subsonic Mach numbers". By that time, in 2006, brief test studies indicated that increased aerodynamic efficiency may be possible for a high aspect ratio wing in ground effect at high subsonic Mach numbers [7], but other analytical treatments suggested the opposite [1]. However, the effects of the formation of shock waves either on a wing upper surface, or between the wing and the ground, were rarely considered in an applied or fundamental context until the most recent decade.

It should be noted that this paper is not concerned with phenomena such as sonic boom interactions with ground objects or water, or shock focussing effects from altitude, as these do not affect the aerodynamics of the body from which the waves originate. Similarly, while the case of a high speed subsonic or supersonic jet impinging on a surface from perpendicular or angled flow is certainly of considerable practical and fundamental interest [8], it lies outside the definition of a body travelling over a surface in close proximity that will suffice for the present work.

Ground effect is commonly categorised in terms of the clearance being within a few characteristic lengths of the ground plane in order for the aerodynamic performance of the body (i.e. an aircraft or vehicle) to be affected. Consider a wing of chord $c$ at a height of $h$, for an $h / c$ ratio of less than 5 (for other bodies a more meaningful characteristic length may be the total length, or diameter). Above this level, the ground has negligible influence, but at lower clearances the lifting performance of the wing is gradually enhanced with closer ground proximity. Extreme ground effect may be taken to mean a clearance that is less than $10 \%$ of the characteristic length of the body. This holds nicely into the transonic and supersonic domain, however, the ability for a body to be non-trivially influenced by a shock reflection from a ground plane at relatively high $h / c$ ratios will later be described for Mach numbers close to 1 .

Basic examples of the kinds of flowfields of interest for this review are shown in Fig. 1 using the example of an aircraft with relevant parameters annotated. The schematics are by no means a full survey of the possible flowfields for high speed ground effect scenarios. Fig. 1(i) shows a typical coalescence of waves from a supersonic aircraft to form a sonic boom felt at the ground, which would produce a characteristic N-shape pressure wave - with significant altitude, the waves cannot reflect back onto the aircraft again or into its wake, and with such distance the waves are also relatively weak. Thus, this flowfield is not considered to be a ground effect scenario.

Fig. 1(ii) presents a case where the ground clearance ratio, $h / l$, may be close to 1 , with the Mach number close to 1 as well. In this situation, local areas of supersonic flow will form and the ground proximity will lead to a ground reflection that may impinge on the aircraft body again, and a ground-related asymmetry in the supersonic region would occur. A photograph highlighting this kind of reflection/interaction is presented in Fig. 2. In such a case, it is likely that a small effect on aircraft aerodynamic characteristics would occur. Fig. 1(iii) shows a supersonic case close to Mach 1 where oblique, near-inviscid reflection from the ground plane may occur depending on the Mach number and shock angle but it is also possible for the bow shock to bend to the wall and an entirely altered flowfield below the vehicle would establish.

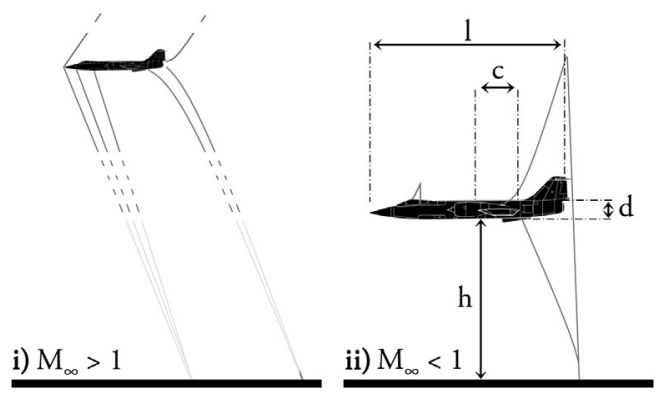

$$
\mathrm{M}_{\infty}>1
$$$$
\mathrm{M}_{\infty} \gg 1
$$

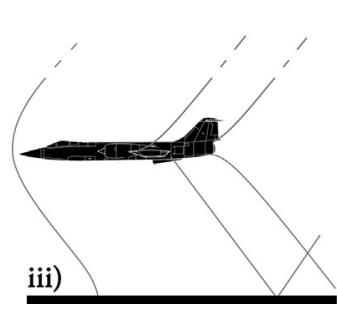

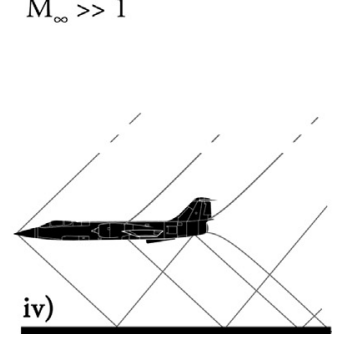

Fig. 1. (i) A high-altitude supersonic vehicle causes a sonic boom at the ground but is not operating in ground effect; (ii) within several height-to-lengths $(h / l)$ at near-sonic speeds, shocks reflect from the ground (other relevant parameters are shown); (iii) at low-supersonic speeds both normal and oblique reflections may occur; (iv) at fully supersonic Mach numbers, one or more oblique shocks may interact with the ground and reflect back onto the vehicle when it is in close ground proximity. 
In (iv) a fully supersonic flowfield features multiple oblique reflections and at a certain low ground clearance these waves would impinge again one or more times on the aircraft, causing significant pressure differentials that would strongly influence aerodynamic performance.

The speeds of the vehicles or freestream flows in question make controlled aerodynamic testing in a conventional wind tunnel a more difficult matter with regards to ground representation, and so in addition to covering the aerodynamic findings relating to bodies at high-subsonic to hypersonic Mach numbers in ground effect, the various means of obtaining accurate experimental data will be discussed.

The focus is therefore narrowed to the following topics:

(1) which methods of ground representation are available to the experimentalist and what level of accuracy do they afford?

(2) what effect does ground proximity have on a body's critical Mach number and what are the consequences of this (i.e. early transonic drag rise, buffeting flow, loss of aerodynamic efficiency)?

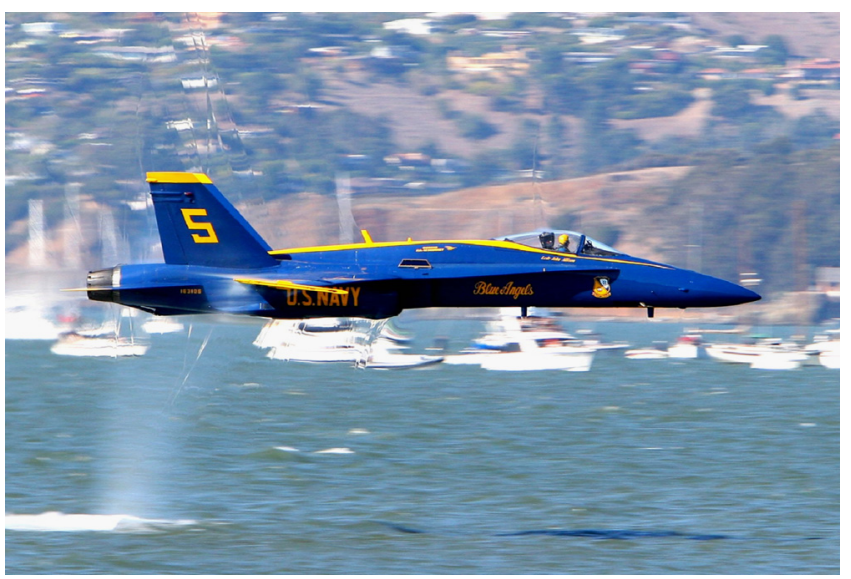

Fig. 2. A US Navy "Blue Angel" demonstration F/A-18 performing a pass at approx Mach 0.95 ( $h / l$ approx. 0.5, mean wing $h / c$ approx. 3). Photo: Matt Niesen (with permission).
(3) what influence does the reflection(s) of shock waves from the ground plane back onto a body - one or more times - have on the aerodynamic characteristics of that body?

A comprehensive overview of studies relevant to these questions has not previously been collated, partly because of the diverse range of sources, the sporadic reporting of activity in the field, and many inconsistencies in terminology across potentially useful works. Some of the research reported in this paper has, until relatively recently, not been widely or publicly available despite it being conducted in the 1960s and 1970s, due to the classified/ military nature of the investigations at the time. Therefore considerable space is devoted to describing the findings of that era, and linking them to the most recent developments in the field; the power of in the 21st century high-performance computing has facilitated a greatly expanded knowledge of high speed ground effect flows through new simulation and re-evaluation of prior data. The review concludes by outlining remaining challenges and opportunities in the field.

\subsection{On shock reflection phenomena, in brief}

A brief outline is provided here of the kinds of shock/surface interactions which are discussed in the following sections; relevant analogies from more fundamental shock wave research are introduced in the process.

Ernst Mach pioneered studies of shock reflection phenomena, publishing a paper in 1875 on the planar shock-wave reflections over straight wedges [10]. He presented two possible wave configurations: a regular reflection, and an alternate configuration that was later named Mach reflection. Fig. 3(i) and (ii) shows basic schematics of oblique reflection configurations: most interest in the ground effect context are flowfields in which both nominally inviscid and viscous reflections occur, and thus consideration of several reflection possibilities is useful. The normal shock with a lambda $(\lambda)$ foot boundary layer interaction is a common occurrence in transonic flows involving wing sections, whereas the supersonic oblique reflections are often studied more fundamentally outside of a direct aeronautical context, but with applications i)

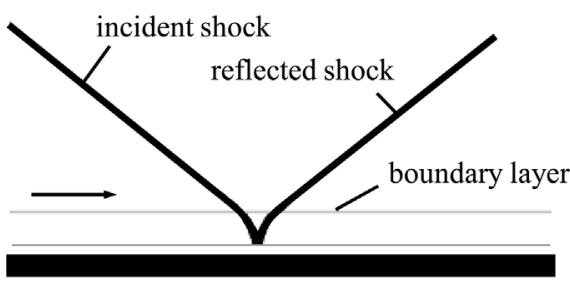

iii)

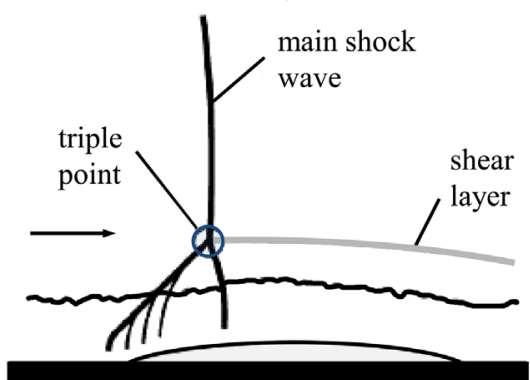

ii)

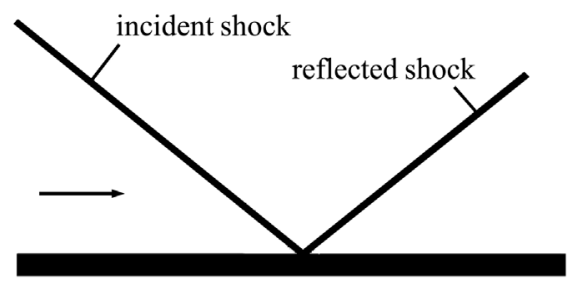

iv)

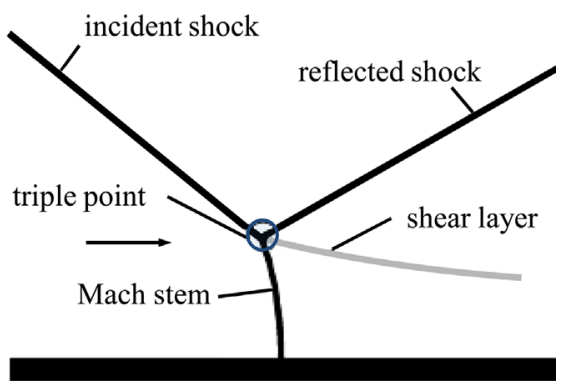

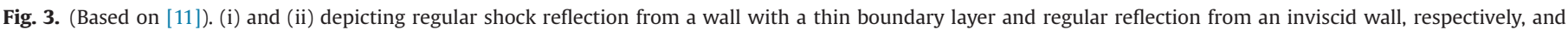
(iii) and (iv) showing normal shock/boundary layer interaction with a lambda shock, and Mach reflection from an inviscid wall, respectively. 
to complex wave interactions on inlets and other aircraft components. Fig. 3(i) shows regular reflection of an oblique shock wave in a fully supersonic flowfield, where a boundary layer is present on the reflecting surface. In the thin subsonic region next to the wall, the shock wave cannot be sustained. Within the boundary layer, the shock and its reflection curve in refraction to normal at the wall - without a boundary layer (ii), in the simplest case, the reflection angle is the same as that of the incident wave. Fig. 3(iv) shows a scenario in which the Mach number is such that a normal Mach stem forms between the incident shock and the ground, and a so-called "triple point" exists at the location where the stem meets the incident and reflected wave. In this case, a shear layer forms behind the triple point. The Mach number and shock angles at which the transition from regular to Mach reflection occurs has been the subject of much debate and research due to difficulties in adequately producing the behaviour in wind tunnels [12]. Fig. 3(iii) shows a typical transonic shock boundary layer interaction, where the foot of the normal shock provokes some separation of the boundary layer and the "lambda" structure forms to reset the flow direction to normal after the disruption of the separation bubble. Behind the shock structure the flow is subsonic, and the main shock is often referred to as the terminating shock. Such waves are prone to oscillation on the surface over a range of high subsonic freestream Mach numbers, which in an aeronautical context is referred to as buffet.

At transonic and supersonic Mach numbers, the formation and reflection from the ground of normal and oblique shock waves is often highly analogous to several other distinct fields of aerodynamic research, most notably wind tunnel wall interference (where the effect is exclusively undesirable and extremely wellstudied [13]), and studies of external stores on aircraft, which have in the past been investigated as simple supersonic streamlined bodies next to a solid surface [14]. In the latter case, when examining the transition from regular to Mach reflection of a reflected wave from a store, the assumption of inviscid flow was deemed to be a minor inconvenience. Ironically this actually serves to more closely approximate the moving ground interactions seen for the supersonic projectile in ground effect studies described in Section 4 of the present work than would have been the case if a fully viscous simulation were feasible at the time. Generally, more recent studies of stores consider cavities, complex on-wing configurations and other fully viscous setups that are far less analogous to high speed ground effect.

In 1986 Hornung stated: "the subject of shock reflection is so complicated that it is necessary to introduce it at some length" [15], and in this spirit the reader of the present work is assumed to be familiar with the basic aspects of shock waves, and is directed to comprehensive reviews of shock reflection and boundary layer interactions available in literature for discussions which do the topic justice, for instance [15-18]. Further discussion of relevant and related material (such as studies involving crossing shock waves) is limited to appropriate points in the forthcoming sections.

\section{Development of experimental methods}

The most pressing, largely unsolved issue surrounding high speed ground effect investigations has been how to undertake experiments. The following section explores the variety of approaches taken and their relative merits. Note that for the purposes of this paper, a standard has been adopted in figures (where possible) in which the flow travels from left to right towards a stationary object (as is customary in aerodynamics for a windtunnel frame of reference), though there are several unavoidable exceptions. For consistency, objects moving through quiescent air are similarly oriented (moving through the image from right to left), though at first this may seem counter-intuitive. Depending on the most appropriate length scale for a given application, the nondimensional ground clearance of objects as a ratio of height, h, is given as $h / d$ (diameter), $h / l$ (length) or $h / c$ (mean wing chord).

The correct ground boundary condition for all ground effect testing in a wind tunnel is a moving ground, conventionally achieved at low subsonic Mach numbers by having a belt travelling at the freestream flow velocity [19]. For larger tunnels and models this is often approximated more crudely with an elevated ground plane that produces its own boundary layer which, though undesirable, can be further minimised with a combination of suction and blowing. In the earliest days of ground effect research, it was also shown analytically that a symmetry (or mirror-image) method can be a good approximation for the flowfield [20]. It has since been discovered that at small ground clearances this can produce inaccurate data [21-23]. High speed ground effect studies similarly require a moving ground, but this is essentially unfeasible from a mechanical perspective for transonic and supersonic Mach numbers.

It is worth visiting some of the findings of studies into ground representation at low subsonic speeds in order to inform the discussion of their usefulness at higher Mach numbers. In 2002 a numerical study highlighted the difference between stationary, slip wall, symmetry and moving ground boundaries for a lifting NACA 4412 aerofoil in ground effect [22]. The symmetry method was seen to produce near-identical results to a moving ground simulation up until very low ground clearances $(h / c<0.05)$, at which point a spurious recirculation ahead of the wing at the ground plane produced somewhat inaccurate results. An analogy was drawn between this observed effect and a vortex pair in a potential flow. The symmetry method did, however, correctly predict a slight liftloss at the lowest clearances, as was observed for the moving ground cases. The CFD was conducted at a freestream Mach number of 0.32 with the flow treated as incompressible, although at this Mach number and at such low ground clearances, one would expect compressible effects to be present.

All the problems of aircraft or road vehicle ground effect testing in wind tunnels are also present for experiments on other vehicles such as trains. However, the additional complication of flow compressibility makes Reynolds and Mach scaling extremely difficult, particularly due to the length of the trains. Studies have shown between $10 \%$ [24] and 30\% [25] error in the values of drag coefficient obtained with scale wind tunnel models and actual track-tested trains. The latter study also noted up to $10 \%$ difference in lift and drag for wind tunnel studies using a moving ground as compared to an elevated ground plane. Baker [26] also indicates that for a large body, a simulation of the atmospheric boundary layer should also be present in the wind tunnel in order to approximate the forces and moments on full-scale trains.

The symmetry method (or mirror-image method) is not commonly used experimentally for any type of body because of extra cost, complexity and tunnel blockage, although the method was implemented for studies by NASA in the 1960s [27]. It was asserted that tests with a symmetry wing setup, with endplates and far from wall interference, had previously produced results which agreed well with data for wings actually moving over a ground, though no specific citation is given. The freestream Mach number was not stated.

As previously mentioned, it can be useful to view high speed ground effect as having much in common with wind tunnel wall interference. In traditional testing, this is unwanted and considerable effort has been expended over many decades to eliminate these effects - one could therefore conceivably find an overwhelming abundance of historical test records in which transonic tests were compromised by wall effects and shock reflections, flow choking, resulting model vibrations, etc. In the case of ground effect studies, these effects are inherently part of the flowfields being investigated, 
and warrant specific attention. While it would be tempting to explore the tunnel wall interference scenario for relatively high ground clearances, it is important to note that the nature of ground representation in the wind tunnel is a signature issue in the investigation of transonic and supersonic ground effect problems. Whereas a wind tunnel can develop an extensive boundary layer on the wall, or have that boundary layer controlled via blowing, suction, bleed to a plenum through holes or perforations, or broader flowfield corrections be applied with adaptive walls [13], a static-model ground effect problem calls for a different approach. Were the tunnel boundary layer adequately removed by such conventional means, a supersonic flowfield would produce wave disturbances generated by any slots, holes or perforations in the surface, potentially interfering with the results. At lower velocities, such disturbances may be even more significant for very low ground clearances.

Since the ground representation and flow behaviour in the vicinity is crucial, the development of a suitable wind tunnel method is vital, and there exists an increasingly important synergy between CFD and experimental work in the absence of any one ideal solution $[28,29]$.

Selescu [30] presents the application of a moving belt system to an ostensibly trisonic (up to Mach 1.4) intermittent blowdown tunnel, yet concedes that its operation is limited to a speed of $111 \mathrm{~m} / \mathrm{s}$, equivalent to Mach 0.33 . Though the mechanism is cleverly synchronised with the blowdown operation, it would not be necessarily more accurate to any worthwhile extent to implement a moving belt at a speed considerably lower than the freestream Mach number.

Recent Japanese development of a high speed jet-driven and rocket-driven subsonic test track of $300 \mathrm{~m}$ in length was aimed at being able to realistically study the behaviour of re-usable space craft in early takeoff and landing phases, both subject to considerable ground effect [31,32]. The maximum speed of the sled arrangement, $200 \mathrm{~m} \mathrm{~s}^{-1}$ (with a lower suggested operating speed of $150 \mathrm{~m} \mathrm{~s}^{-1}$ ), equates to approximately Mach 0.6, placing it within the realm of strong compressibility effects between the craft and the ground, but unlikely to reach the critical Mach number unless an object were placed in extreme ground effect. As this facility is only recently operational at the time of writing, no detailed test results on aircraft/spacecraft configurations have been reported but could be expected to be of great interest in terms of ground effect behaviour close to the transonic regime. Despite the obvious considerable expense, the advantage is clearly that of considerably higher Reynolds numbers than could be obtained even in a sizeable wind tunnel, and the ability to better study acceleration and deceleration phases as no formal, controlled, thorough aerodynamic study of these phases has been reported for any application to the best of the author's knowledge.

Rocket sled facilities for higher speed applications (transonic, supersonic and hypersonic testing) are generally military property, and well established in many countries. In brief, as shown in Fig. 4 (i), based on the test work of Strike and Lucas in 1968 [33], the common design involves a test vehicle propelled along steel rails by (often multi-stage) rocket motors, guided by a slipper which wraps around the rail to provide stability and restraint. The relative merits for performance and stability of various rail gauges and arrangements are summarised by Minto and others [34] and are not important for the present discussion.

Studies using rocket sleds are almost exclusively related to nonground effect applications, and the goal of researchers is to place the test object out of ground effect even though the rocket sled itself operates in such conditions. Nevertheless, research related to the design and development of such systems offer greater insight for the current topic. Several studies into the evolving design of the Holloman facilities in New Mexico were conducted during the 1960s and 1970s [33,35], and while similar sleds exist around the world Holloman is exceptional for the amount of research publicly available on its design and development. Wind tunnel testing of Fig. 4 sled arrangement was conducted from $M=1.5$ to 4 , with an elevated ground plane. Results were shown to agree well with subsequent tests with the actual rocket sled, although the margin of error stemming from poor repeatability of ground clearance was considerable. The differences in setup from wind tunnel to real-life test are shown in Fig. 4 - this also highlights the issues faced in the wind tunnel, including interference from the elevated ground leading edge, bow wave/ground BL interaction, and a differing boundary layer velocity distribution between sled and rail.

In the wind tunnel tests, it was noted that the normal force and pitching characteristics of the model (which was a 1/12th scale cylinder with a conical nose mounted to a rail above the elevated ground) changed sign with increasing Mach number before and

a

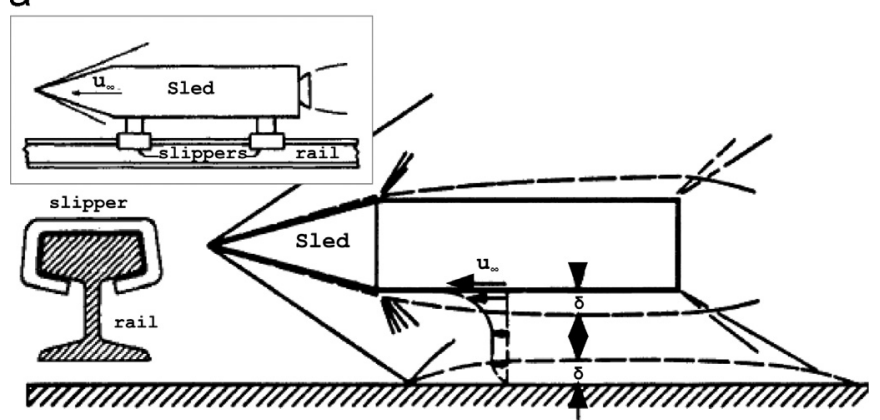

b

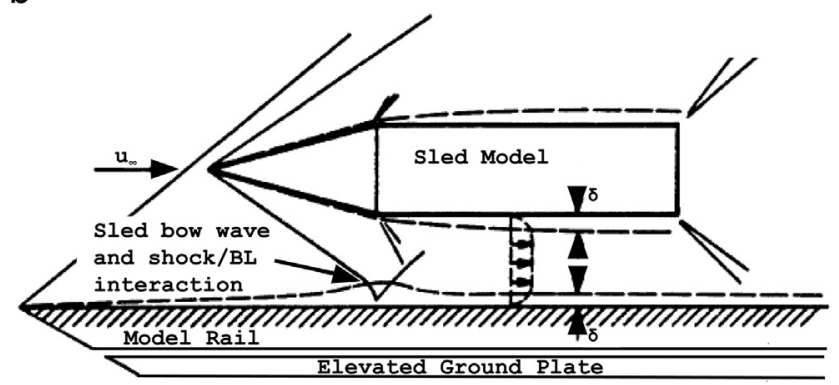

Fig. 4. Schematic of a rocket sled mounted to a rail above the ground for (a) realworld conditions (actual track environment) and (b) a scale model in a wind tunnel using an elevated ground plane (wind tunnel conditions). Adapted from: [33].

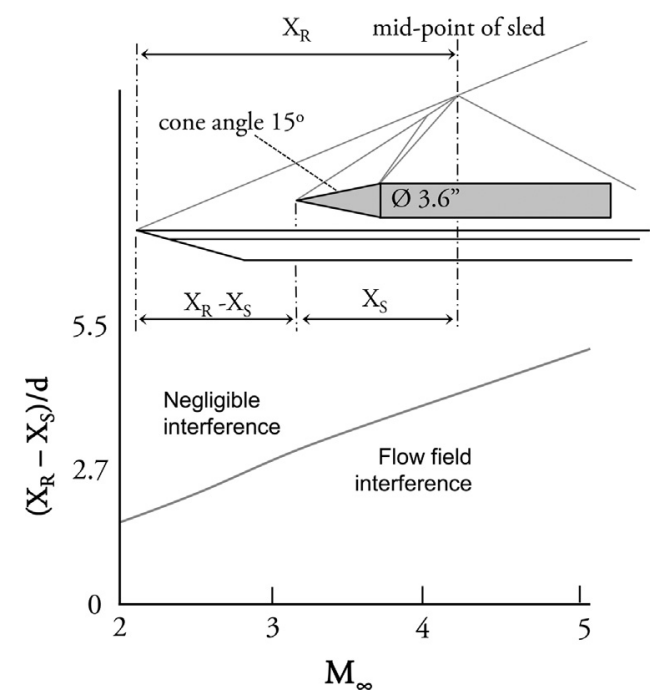

Fig. 5. Wind tunnel tests for a basic rocket sled/slipper/rail arrangement using an elevated ground, indicating likelihood of flow interference from the leading edge of the ground plane.

Adapted from [33]. 
after $M_{\infty}=2$. Although not specifically noted in the report of Strike and Lucas [33], this would most likely have been a function of the way in which the shock from the nose was reflecting from the ground plane and interacting again with the model in relation to the centre of gravity. Rather than consistent trends, the normal force acting on the model was observed to increase and decrease considerably across the Mach number range tested, indicating complex shock interactions from the simulated rail as well as the elevated ground plane, including downstream influences on the model sting and force balance due to multiple reflected shocks these interactions will be discussed further in Section 5 .

Strike and Lucas also spent considerable time evaluating the rail tip geometry for wind tunnel tests [33]. In real sled tests, the rail is effectively infinite, but in the wind tunnel the replica must sit above the elevated ground plane. Therefore the flowfield includes not one but two shock/ground interaction problems, as the upstream tip flow determines how the air meets the sled body downstream and is somewhat sensitive as a result.

Fig. 5 indicates the relationship which can exist between the leading edge of an elevated ground plane and the model. In order to avoid unwanted wave interference on the body being studied, the ground plane must extend upstream by a certain distance that is related to Mach number - due to shock angles - and the length of the body. In some instances this may mean a considerable ground run upstream which may prove difficult to mount effectively, and which introduces a significant boundary layer that may almost defeat the purpose.

Sled testing itself - clearly the most preferable option for high speed ground effect testing were it not for expense and scarcity raises two distinct challenges. Firstly the test object (as opposed to the sled rocket), be it a missile or other payload, should preferably be free of any shock reflection such that its aerodynamics may be assessed as an accurate representation of freeflight, However, placing the test object either too far forward of the booster rocket and support structure, or too high above the rail, could result in prohibitively high moments that would otherwise demand an unfeasibly strong and durable structural requirement (particularly when aerothermodynamic loads are exceptionally high). Commonly, for supersonic and hypersonic velocities, the test object is therefore close to the ground and closely linked to the main sled apparatus, placing it in close proximity to the guide rail and often the ground itself. Secondly, the design of the actual rocket sled itself, regardless of the location and nature of the payload, must take shock reflections and interactions into account, both for their effect on the aerodynamic loads of the sled that may lead to complex aeroelastic coupling [36], and their potential for thermal-induced fatigue due to shock impingement on parts of the apparatus [37].
In the case of aeroelastic coupling, some design aspects are of particular concern - vibration and excitation of the rail and/or the sled due to shock impingement and transient loads imparted as a result, and the tendency for the sled and payload to want to lift and pitch due to the build up of high pressure underneath as a consequence of multiple shock reflections (a defining characteristic of supersonic ground effect flows). The fact that the sled may accelerate rapidly through an unstable regime of force and pitching changes exacerbates this issue.

The study of Lamb [36] details the necessity to understand the influence of shock reflections back onto the rocket sled test bed of the Holloman tracks. Structural failures resulting in payload loss at the facility due to vibration issues prompted an investigation of the potential for shock reflections and interactions with the rail and slipper geometries. At the 10 mile Holloman High Speed Test Track, regularised sled-rail impact frequencies were measured. While some inconsequential frequencies may be attributed to vortex shedding, the reflection and interaction of the bow wave with the periodic tie-downs holding the track in place and assisting with rigidity and alignment were shown to produce resonance-mode feedback and the assumption of a purely rigid rail was shown to be incorrect. Fig. 6 reproduces an image of the so-called "SM-2" payload close to Mach 4 and has been annotated with approximate shock cone shapes representing Mach 1.87 and Mach 2.56 conditions; a pressure transducer was located at the position denoted $\mathrm{P}$ and the rail is denoted by the two parallel white lines - the potential for shock reflection and re-impingement on the payload from the tie downs, represented by the white box, is evident. Fig. 6 also reproduces the frequency analysis from the test, indicating the shock reflection effect across the entire available supersonic Mach number range.

That study concluded by stating that a more holistic approach, integrating consideration of the "interplay among the sled weight, velocity, aerodynamic forces, and the resulting sled-rail impact force is essential" for allowing more aggressive testing into the hypersonic range without such a high risk of rail fracture and fatigue. It is likely that the role of CFD in such studies could be greatly expanded, since physical testing is an expensive and risky pursuit - a high resolution model could help identify shock behaviour at a wide range of Mach numbers in a dynamic or quasi-dynamic environment, with LES-based methods able to assist in distinguishing vortex-shedding frequencies with reasonable accuracy. A more complex fluid-structure interaction approach would be likely to yield valuable predictive insight into rail and sled interactions.

Such a simulation-based path has been started down by researchers such as Turnbull et al. [38] who more recently
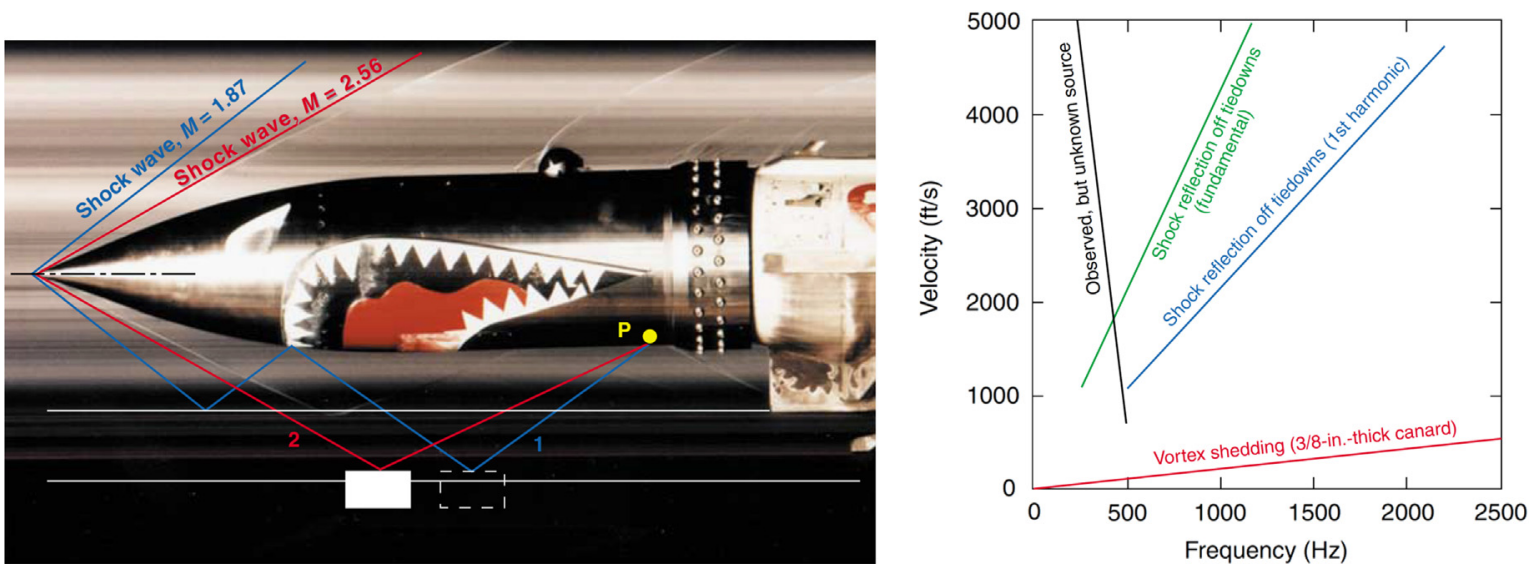

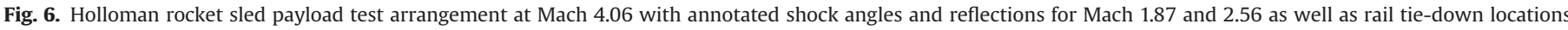

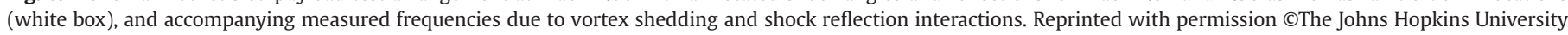
Applied Physics Laboratory.

Source: [36]. 
reported on the ongoing development of "soft-sled" capabilities at Holloman that are designed to lessen the severe vibration environment. However, it is worth noting that that analysis was conducted with an Euler solver that would not produce the more complex shock/BL interactions which characterise shock reflections back onto the sled geometry, and this may influence the structural response predicted by simulations. Similarly, a report on design software developed specifically for rocket sled development, as reported by Hegedus et al. [39] promises reasonable, rapid first-pass accuracy with an Euler solver but as observed with the high-resolution viscous simulations reported by others [40], even a slight miscalculation of shock re-impingement location on a curved forebody could result in a significant over or under estimation of loads for a given Mach number, and surface heating effects due to shock/BL interactions into the hypersonic range would require more advanced modelling anyway.

In order to perform a detailed comparison between wind tunnel experiments using an elevated ground plane and realworld sled performance, another set of tunnel tests were conducted in 1967 across a Mach range simulating acceleration and deceleration of different sled configurations [41]. Both vertical wedge-based noses, "arrowhead" shapes and combinations thereof were examined. An example of the results obtained can be seen in Fig. 7 for the "GNU sled", which was a relatively thin vertical wedge nose (with a blunted tip), supported by two rocket boosters on either side towards the rear of the 5.2 m body, running on a dual rail. Different bodies produced markedly different forces, but all featured a strong reaction to the transition from transonic
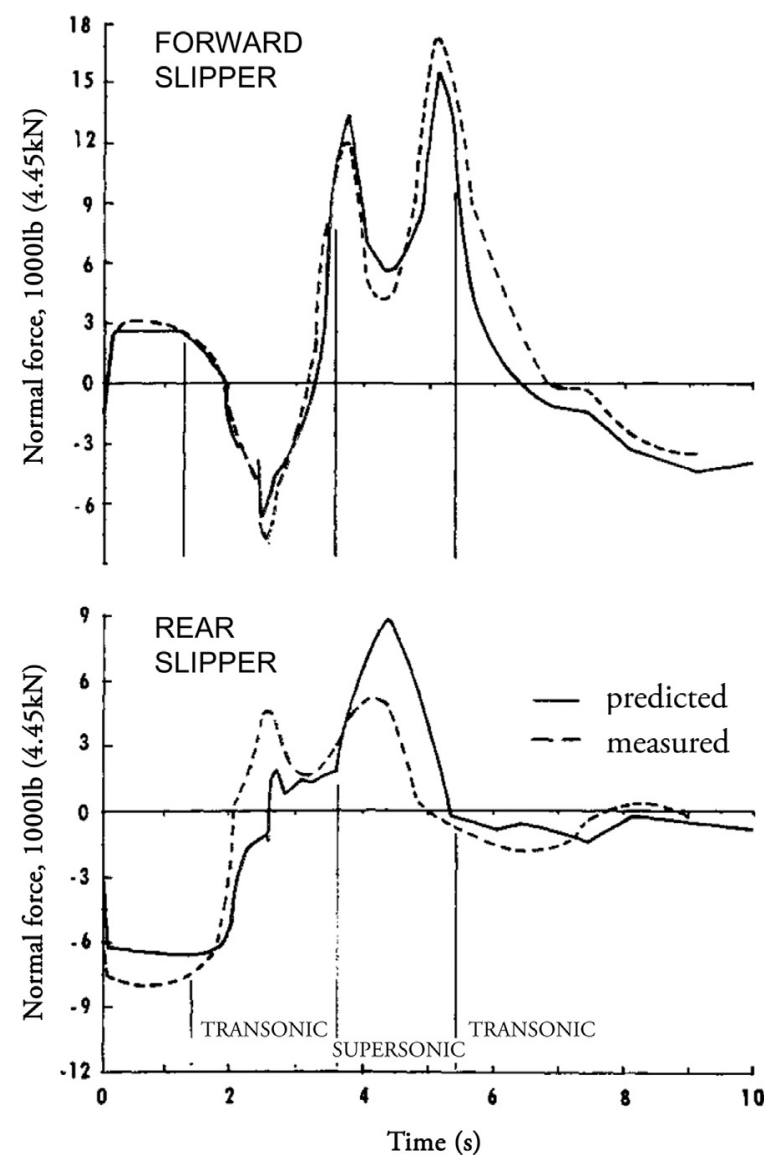

Fig. 7. Rocket sled front and rear slipper loads during acceleration and deceleration phases through Mach 1; comparison of real-world test and interpolated predictions from wind tunnel data.

Adapted from [41]. to supersonic Mach numbers - the causes for such effects will be discussed further in Section 3. The wind tunnel predictions and the actual measured force values from the sled are similar in trend but different in peak magnitude for the forward slipper. At the rear slipper, the predicted forces are more significantly offset -this point is after the first series of shock/ground interactions and the nature of that interaction in the wind tunnel, with a ground BL and geometric simplifications, may be responsible for the discrepancies as well as Reynolds number effects [33].

It is also apparent that there are large differences in forces between the front and rear slippers particularly where lift changes sign, indicating potentially large swings in pitching behaviour as well. The peak lifting force occurs in the supersonic regime whereas transonic flows are generally characterised by downforce - at least up until shock waves form in the accelerating phase where a lower surface shock may have been responsible for the front downforce in particular. Intriguingly, as the sled transitions back to transonic Mach numbers after $5.5 \mathrm{~s}$ of a real sled run, the forces do not follow the same trace as observed in the accelerating phase, pointing to a strong transient phase in either direction where no quasi-steady flow is obtained [42].

This transient phase was also noted in passing by Carriage et al. [43] in examining a small projectile in ground effect at a Mach number of 1.2. The schlieren images were resolved enough in time and space to discern a continuing adjustment period of shock strength and location following the projectile first passing over the surface. The time taken to reach a nominally steady-state was not quantified but noted as a point requiring considerable further investigation as the location of measurements on a ground plane would have to be guided by this adjustment phase. The reflection of the waves and their subsequent interaction with a spinning boundary layer on the projectile is a unique situation in aerodynamics which is addressed in subsequent sections - for this particular problem, the inability to reproduce the high spin rate in scale wind tunnel tests was investigated with CFD and found to be a very minor influence [40].

Korkegi and Briggs [44] note that at very high speeds the aerodynamic lifting forces on a rocket sled may exceed "by far" the dead weight of the apparatus, leading to extensive metal-to-metal contact on the underside of the rail as the slipper is pulled into it. Again a product of the build up of high pressure underneath the system constricted by proximity of the test object to the rail and ground, this obviously leads to severe friction in addition to aerodynamic heating. Intriguingly, the slipper itself operates in exceptionally close proximity to the rail in its own "ground effect", and boundary layer flow behind the local bow shock is forced into the fluid gap to create a Couette-esque aerodynamic effect. The pressure in this gap decreases with gap height (analogous to ground clearance reduction) rendering the configuration as statically unstable and prone to track-gouging.

To counteract the tendency towards extreme lifting behaviour, canards are commonly attached to the nose-cones of rocket sled forebodies to provide a measure of counteracting downforce, and these are also routinely attached to the forebodies of similarly shaped rocket or jet-powered land speed record vehicles such as the Budweiser Rocket, the Bloodhound SSC, etc.

Predating the modern era of CFD, a handful of land speed record cars were designed with the aid of a wind tunnel - the much-revered Goldenrod streamliner, which held the wheeldriven land speed record from 1965 to 1991 (and a nonsupercharged record until 2010), was developed using the CalTech $10 \mathrm{ft}$ tunnel with a $1 / 5$ th scale model [45]. The tests utilised an elevated ground plane with significant upstream reach and a gently contoured leading edge, and the chief aerodynamicist of the project reported that the wheels were elevated above the ground by the estimated thickness of the boundary layer to chase extra accuracy. While this may have been partially effective, the 
boundary layer responds to the local pressure field and therefore would not react as a consistent quasi-surface in the vicinity of a vehicle. It has also been reported for subsequent studies on wheel aerodynamics, albeit for open rather than enclosed wheels, that any gap between the bottom of the wheel and the ground may result in extremely inaccurate, or even reversed, force predictions for that wheel due to the lack of a high pressure spike in the contact patch region [46].

The electric land speed record vehicle Buckeye Bullet 3 is described by Bork [47] as being subject to extensive CFD development that in turn builds on wind tunnel work on the Buckeye Bullet 2 at the Penske facility in North Carolina, where a 1/3 scale model was tested. That model featured no wheels or underside detail; a facet which the CFD later revealed to be significant, with wheels accounting for over $20 \%$ of the vehicle drag even when almost completely covered, making a compelling case for a close synergy between CFD and wind tunnel to better understand the limitations of both. Although the CFD was run for speeds up to $500 \mathrm{mph}$ (in the Mach 0.7 vicinity), no mention is made of any supersonic flow in the study.

The Blue Flame rocket-powered land speed record car - the first vehicle explicitly designed and built to be likely to go supersonic on land - was developed with a $1 / 25$ th scale model in the Ohio State University transonic wind tunnel with a $12^{\prime \prime}$ by $12^{\prime \prime}$ test section featuring perforated walls [48]. The researchers indicated a consideration of the possible benefits of a symmetry model in the tunnel, but in the end pursued an elevated ground for the test programme. The ground was pressure-tapped to check for flow separation on the ground plane. This potential to separate the ground boundary layer with a sufficiently strong incident shock would then produce an induced separation shock and a re-attachment shock affecting the body downstream [49]. These altered reflections would influence the accuracy of any results obtained, further supporting the case for ground boundary layer minimisation as a percentage of the ground clearance and model size.

Detail was not given with regards to the design of the leading edge of the elevated ground. Schlieren images, though difficult to discern from, indicate that at the rear of the vehicle in the vicinity of the proposed wings, the boundary layer on the elevated ground occupied approximately $33-50 \%$ of the vehicle ground clearance [48]. However, this visual appearance is likely to be exaggerated in the image due to the image's effective integration of the flowfield across the section including the boundary layer at the ground/window junction. However, the boundary layer would have distorted the pressure and force readings taken. Images for Mach 1.1 runs also appear to show tunnel wall interference. Measurements made on the actual vehicle indicated that the drag predictions of the wind tunnel model were up to $40 \%$ higher [50], and it is likely that this boundary layer interference with the flow would have been a considerable contributor to this. The authors note that "only local separation of the boundary layer on the plate occurred" but it would not be known how realistic this was compared to the real world, as the wind tunnel boundary layer would be undoubtedly thicker.

To date the only effective synergy between experiments and CFD for a transonic ground effect vehicle has been the Thrust SSC car, which was the first, and so far only, to set an official supersonic land speed record $(M=1.0175)$. When testing the concept of the Thrust SSC supersonic land speed record car, designers were able to conduct tests using the Pendine Sands rocket sled facility in Wales [51]. The Pendine Sands rocket sled is $1500 \mathrm{~m}$ in length with a maximum capability of Mach 3 [52]. The small-scale model was pressure-tapped, as was a specially implemented flat ground section over which the model passed at a lowest "wheel" clearance of $1 \mathrm{~mm}$ [53], over 13 runs and speeds of up to $820 \mathrm{mph}$ $\left(367 \mathrm{~ms}^{-1}\right.$ ). Haddleton describes the high-speed photography of these $1 / 25$ th scale model tests [54] and the use of the imagery to establish the floor ground clearance, quoted as being $25 \mathrm{~mm}$ and $13 \mathrm{~mm}$ on two occasions. Though difficult to discern due to some motion blur, shock interaction with the ground is visible. The data were never published, but the agreement between experimental results and CFD conducted at the time was deemed to be excellent [53,51]. However, the CFD, while state-of-the-art in 1992/1993, featured a markedly simplified geometry (including symmetric rear wheels instead of the offset, staggered arrangement used on the final vehicle), and the fluid was treated as inviscid. Given that the boundary layer on the test model would have been considerable in relation to the model itself, and numerous underbody shock-boundary layer interactions would have occurred, such agreement may have been fortuitous to an extent.

Examples of such rocket sled usage to specifically examine high speed ground effect are exceptionally rare compared to the use of small wind tunnels. The basic premise, problems and potential of both symmetry and elevated ground methods in a blowdown supersonic tunnel are further highlighted by the colour schlieren images in Fig. 8. The model used for these wind tunnel experiments was a simple wedge shape; an extruded two-dimensional geometry with a horizontal undersurface. The upper surface was a double-angle wedge, at $22.6^{\circ}$ from the leading edge, reducing to $5.1^{\circ}$ on the top surface. The experiments were performed in the supersonic blowdown wind-tunnel of the University of New South Wales at the Australian Defence Force Academy (ADFA) campus. The facility was fitted in this instance with a Mach 2 liner. Two ground clearances were investigated: $5 \mathrm{~mm}$ and $10 \mathrm{~mm}$, which equate to 0.0625 and 0.125 height-to-length $(h / l)$ ratios respectively.

The presence of a shock followed by an expansion on the lower side of the leading edge denotes that the leading edge is not quite a perfect sharp edge, and that the flow structure is still highly three-dimensional when the object has a low aspect ratio, creating a shock cone that perpetuates around the entirety of the model. It may be assumed that the leading edge shock from the underside is extremely weak given the lack of a definite flow-turning surface, although its exact strength is difficult to determine from schlieren alone. One can also see the point at which this cone interacts with the test section window, due to the way in which schlieren highlights gradients in planes perpendicular to the viewing angle. From these images it also becomes clear that the potential for model deflection is problematic as even a slight change in ground clearance can alter the flowfield substantially, as seen in image (c) where the model was sucked towards the ground plane from its initial horizontal installation. While this is likely to be more of an issue for an elevated ground case, care must be taken that the ground itself is well-aligned with the oncoming flow, and that the symmetry models are as perfectly symmetrical as possible. This can be difficult even with good manufacturing tolerances and image (d) shows that a marginal asymmetry in shock patterns can be caused by slight mis-alignment in the vertical plane too.

Following those initial experiments highlighting many of the possible issues, the feasibility of using the elevated ground and symmetry methods for fully supersonic problems was investigated further by Doig et al. in 2008 [40], with a projectile model designed to replicate tests on a spinning projectile (a NATO $5.56 \mathrm{~mm}$ round) that will be described in more detail in Section 4. The projectile's size allowed close Reynolds-scaling (geometric 2.5:1) as well as matching the flight Mach number of 2.4 in the wind tunnel, although the projectile's high spin rate was not deemed to be practically reproducible. The elevated ground plane was a simple design, very similar to those used for the Holloman rocket sled tunnel tests [33], that featured a sharp wedge at the leading edge that left the upper surface parallel to the flow. The symmetry model consisted of two projectiles stung from the rear 

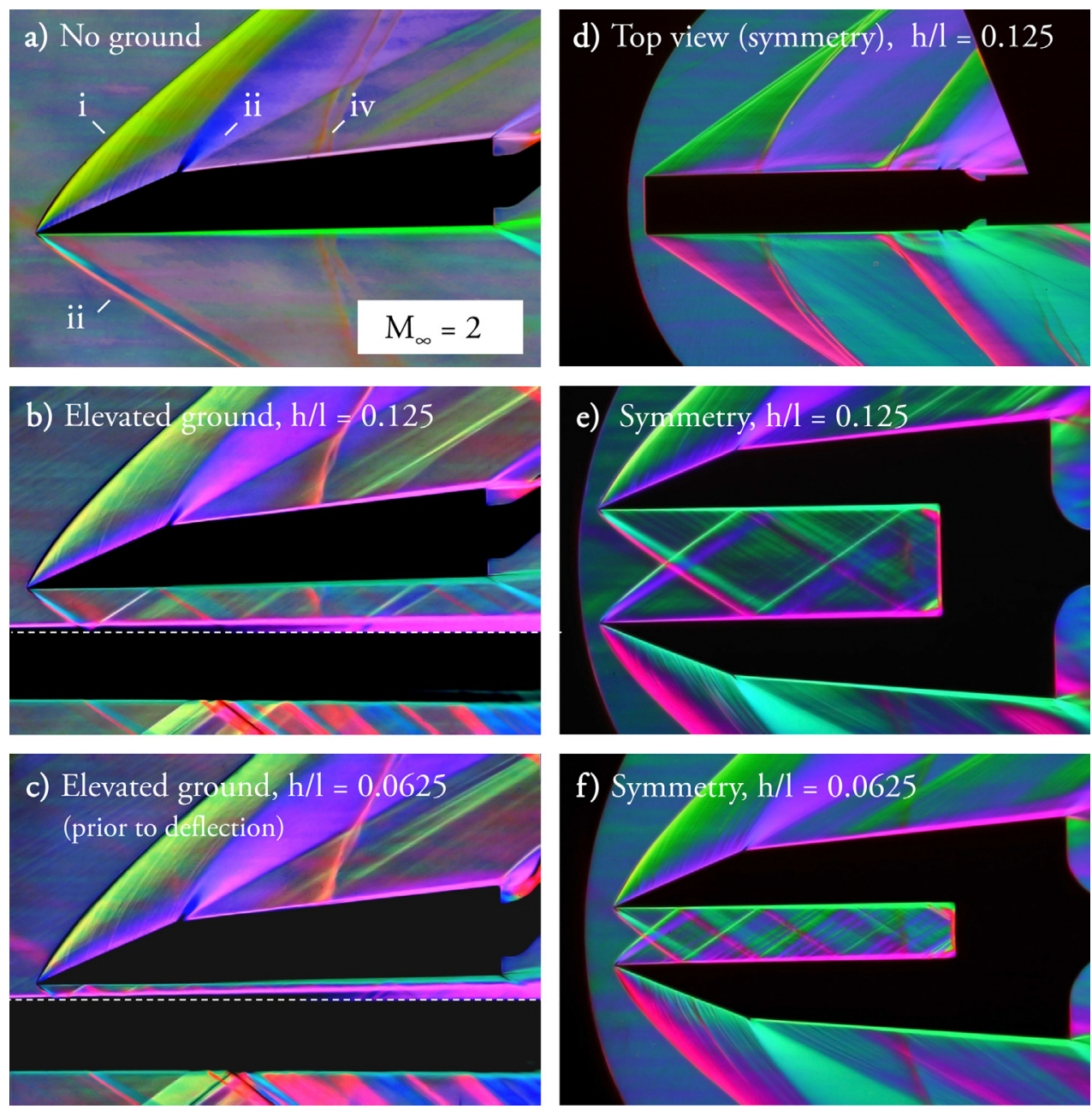

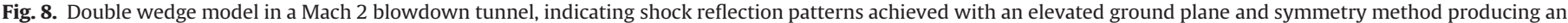
"imaginary" ground. (For interpretation of the references to colour in this figure caption, the reader is referred to the web version of this paper.)

and held at "ground" clearances of $h / d=0.5$ and 0.42 . At the latter clearance, on the elevated ground, the boundary layer was estimated from Schlieren images and analytical calculations to be over $40 \%$ of the clearance space, and as with the double wedge model the shock reflection patterns became notably exaggerated the further downstream measurements were taken - the first shock reflection of the elevated ground plane was 3.5\% further upstream, the second $6 \%$, etc., indicating a propagation of errors with each reflection.

This would assume that the symmetry method was a good approximation of a moving ground, but to make this comparison the authors had to rely on RANS CFD of the wind tunnel experiments. Having validated with good agreement between predicted and measured pressure distributions around the projectile [40], the CFD indicated that the symmetry and moving ground results were essentially identical in terms of projectile surface pressure distribution and forces, whereas the elevated ground plane offered the expected offset and slight diffusion of the reflecting shock wave. No comment was made on additional influence from the ground plane leading edge.

The $h / d=0.42$ case was highlighted as being of particular interest as the elevated ground produced a reflected shock impingement that coincided directly with the expansion region caused by the geometric blend from ogival nose section to cylindrical body, whereas the symmetry method predicted an impingement location just downstream, causing a large difference in local $C_{P}$. Lift force was deemed to be $4 \%$ higher for the elevated ground, with symmetry and moving ground being within $0.1 \%$ of each other (i.e. within the bounds of numerical error), though it was acknowledged that having a physical ground plane allowed for significant instrumentation and visualisation not otherwise possible. Example comparisons of RANS CFD models of these wind tunnel tests, including the $h / c=0.42$ test, are presented in Fig. 9 . While it is apparent that the symmetry method results are nearidentical to the moving ground result in terms of projectile pressures, it is clear that an $h / d$ of 0.2 the ground boundary layer formed on the moving ground is actually influential enough, given the small clearance, to produce a different pressure distribution in the region behind the impingement of the rear lower recompression shock, perhaps even indicating mild separation that is also present in the elevated ground case. However, the elevated ground mis-predicts the shock-impingement $C_{P}$ itself and also exhibits a diffused distribution in the vicinity of shocks on the projectile.

When the symmetry method is used, alignment must be of the highest precision otherwise the flow will become distorted and end up a poor representation of the moving ground flow - slight misalignments have been seen to have obvious and detrimental effects in some reported studies [55], and thus care must also be taken to perform careful measurements of any deflections in the wind tunnel with wind on.

Doig et al. attempted to extend the study of effectiveness of an elevated ground and symmetry technique to unswept wings in a Mach number range from 0.5 to 0.8 [56]. Wind tunnel testing was conducted in the 0.2 by $0.2 \mathrm{~m}$ transonic wind tunnel at the United 

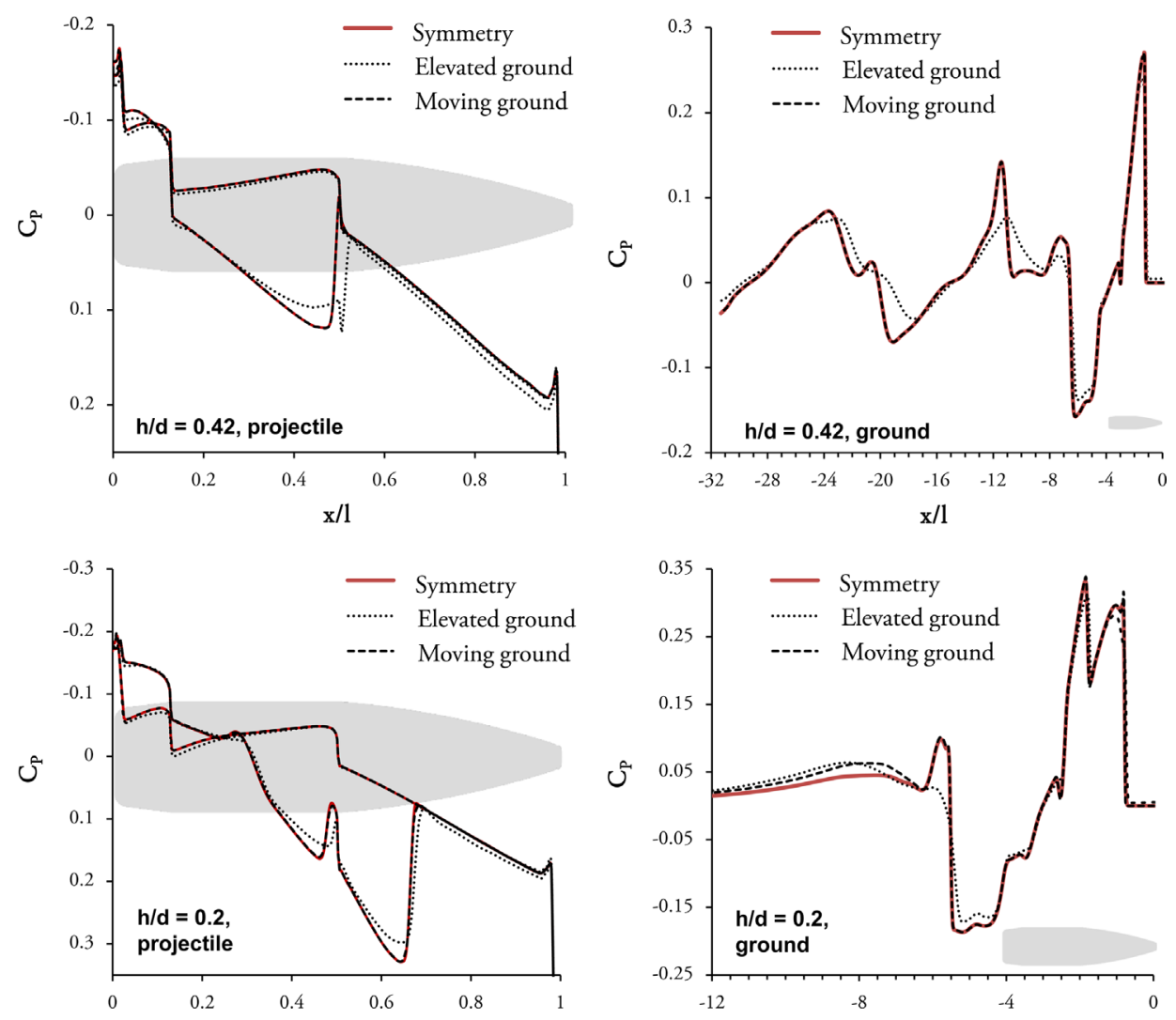

Fig. 9. Pressure coefficients from a CFD replication of wind tunnel tests for a projectile model at Mach 2.4 , comparing ground representation techniques at $h / d=0.42$ and 0.2 .

States Naval Academy and used an RAE2822 wing section of aspect ratio 3, featuring a turbulent trip near the leading edge and staggered chordwise pressure tappings. The authors chose to keep the porous floor and ceiling closed in order for the tests to be easily replicable in CFD, however the inconsistent blockage percentages caused by the two different methods was found to have a non-negligible influence. This led the study to rely more on the RANS modelling as validated against the tunnel experiments than the tunnel experiments themselves. Nevertheless, some pertinent conclusions were drawn: For all Mach numbers and ground clearances investigated, the symmetry method provided a superior match to results obtained from CFD featuring a moving ground.

Only at clearances approaching an $h / c$ ratio of 0.1 did minor discrepancies in the pressure distribution close to the leading edge and suction peak appear. This was partly due to the minor influence of the thin ground boundary layer forming on the moving ground but not on the symmetry axis, and also due to discrepancies emerging as the high gradient region close to the stagnation zone began to merge with the ground and symmetry plane, behaving differently in each case.

Pressure distributions on the wing and ground centre planes in Fig. 10 highlight the fact that without significant additional flow acceleration between the wing and the ground (such as that occurring at low angles of attack), all methods produce acceptable, close results. If the clearance is low enough that boundary layer thickness becomes an issue, or that local supersonic flow may form between the wing and ground, then neither method provides a truly accurate prediction of moving ground flow, with the symmetry method unable to incorporate the slight alteration to the effective cross-sectional area caused by the boundary layer which forms on the moving ground, and the elevated ground greatly exaggerating this effect.

The elevated ground had a tendency to artificially increase the effective angle-of-attack of the wing by deflecting more air over the upper surface. At low ground clearances, the ground boundary layer served to further constrict the flow between the wing and ground such that higher Mach numbers were achieved over both surfaces of the wing compared to those obtained with the symmetry method. Both these effects served to reduce the critical Mach number. It was also noted that the elevated ground approach has an in-built limitation in that when it reaches its own critical Mach number, the downstream flow would be significantly disrupted and bear little resemblance to that which would be attained with the symmetry method. Issues of scale were present as they would be in any wind-tunnel test $[57,58]$, though complicated slightly by the relatively large boundary layer compared to the overall ground clearance compared to supersonic ground effect cases.

One can appreciate that even a better design of the leading edge, featuring a contour which avoids the risk of any separation, has two intrinsic limitations. Firstly it will accelerate the flow at the ground plane to a value which is faster than the freestream. This is particularly problematic if the ground clearance of the test model is small, in which case the lower surface could 'see' an entirely different oncoming flow than the upper surface. Secondly, introducing significant curvature at the leading edge, even if it is designed to settle the flow downstream to a level which would not significantly disturb the wing, would still result in the emergence of a shock wave at a certain Mach number.

Returning once again to aspects of wind tunnel interference for conventional testing, it is possible to find other pertinent insightful data relating to the role of a splitter plate (very similar to the elevated ground principle) for transonic testing - commonly implemented as a means of negating the side wall boundary layer by introducing what is effectively the same mechanism as the elevated ground discussed here. Tests conducted in the NASA Langley Transonic Dynamics Tunnel, reported by Schuster [59], from subsonic to low-supersonic Mach numbers with a side-wall 
a

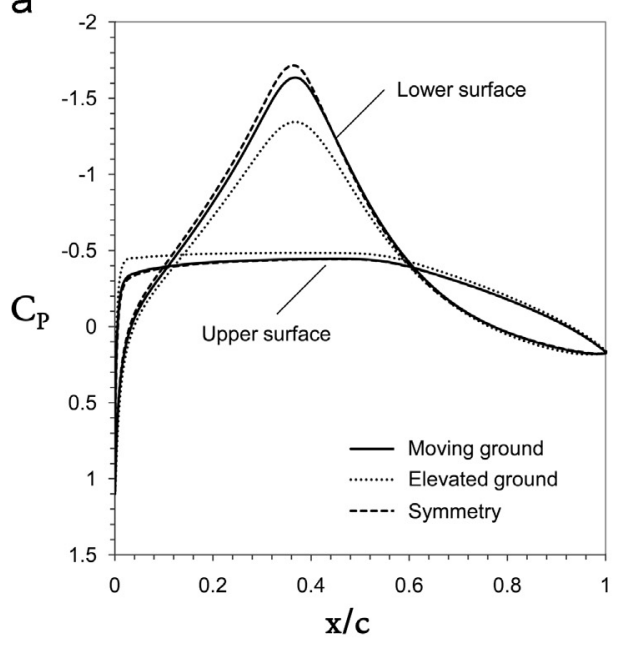

b

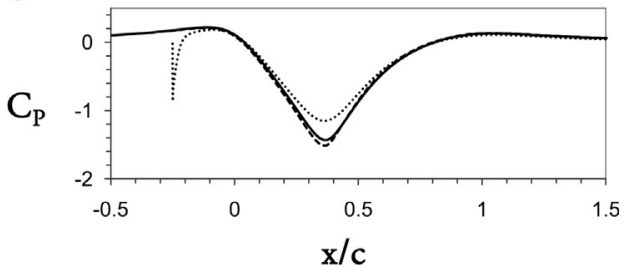

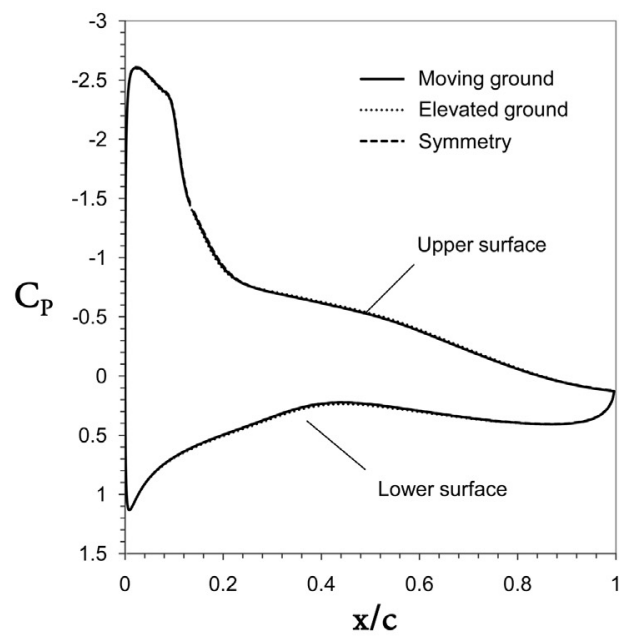

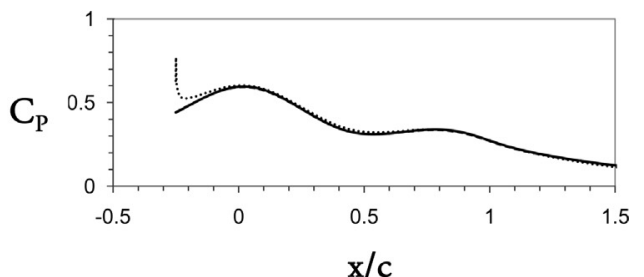

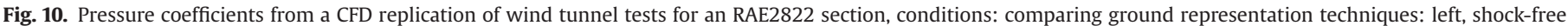
flow, $M_{\infty}=0 \cdot 53, \alpha=0, h / c=0 \cdot 13$, right, $M_{\infty}=0 \cdot 63, \alpha=6, h / c 0 \cdot 23$.

Based on [56].

splitter plate indicate that the flow is relatively free of interference effects until the supercritical stage is reached (between $M_{\infty}=0.7$ and 0.8). The shape of the leading edge of the plate, in particular, was found to be a strong determinant in defining the boundary at which sonic flow occurred. A shock wave near the leading edge of the plate was observed at Mach 0.9, and for low-supersonic speeds the interference is deemed unacceptable. Schuster also alludes to the blockage effect (the splitter plate must have sufficient mass and strength in its mounting and as a result may become bulky) by noting that with the plate installed the upper Mach number limit of the tunnel reduced from Mach 1.2 to 1.05 , and that unsteadiness in the flow from the plate was a concern. As a result, testing above Mach 0.8 was not recommended for anything other than crude qualitative analysis when the plate was in use, although blowing and suction are proposed as being partial solutions to the onset of problematic flow on the splitter plate itself.

While most rocket sled testing involves supersonic and hypersonic velocities, some testing is conducted in the transonic regime. The design of a suitable testing rig for wing and wing/body configurations is described by Krupovage [42], whereby a large "flatbed sled" ground plane was constructed between the two rockets mounted to each rail. A wing would sit at a considerable distance above this plane, in an attempt to be free of interference from the more complex sled structures normally used, with results compared to reliable, corrected same-scale wind tunnel data. With the ground plane parallel to the flow, a small upwash was measured, and thus the ground was tilted down at a "slight" angle. Photographs indicated that the leading edge shape was a curved, sharp ogival-profile, therefore the plane itself would act like a wing in ground effect; the wing model itself was mounted significantly downstream of the leading edge - rather than horizontal however, the wing was designed to be mounted vertically in the fashion in which half-models are commonly mounted from the floor of a wind tunnel, as ground effect was not the actual focus of the study.
While the authors concluded that there was no ground influence on the test model wing model due to it being mounted sufficiently far away, the measurements of the ground plane itself indicate that a shock wave formed and travelled aft of the leading edge with acceleration - furthermore, as previously reported for different tests by the same author [41], the acceleration and deceleration phases produced different pressure readings at the same Mach number. This serves as further evidence that an elevated ground plane will indeed produce disruptive upper surface flow effects above a critical point, and that the resultant shock would be prohibitively disruptive for testing of an actual body in ground effect in the near-sonic region. We may also deduce that the elevated ground itself was operating as a wing in ground effect as it's $h / c$ ratio was significantly less than 1 (from photographs), and the effect of this was unfortunately not quantifiable for the reported tests.

A final illustration of the inability of the elevated ground plane to accurately describe many extreme ground effect flows comes in the fundamental study of Dudley and Ukeiley [60]. A circular cylinder was placed in a Mach 1.44 freestream flowfield but close enough to the wall to be inside the boundary layer. The Reynolds number was between 1.9 and $3.8 \times 10^{4}$ based on the cylinder diameter. The authors note that the wake behaviour was strongly affected by the presence of the wall, exhibiting typical Karmantype shedding behaviour not seen at higher clearances (i.e. the sonic line) away from the boundary layer, and additionally the ground boundary layer was periodically influenced to incorporate its own separation and shedding behaviour. While it is also true that the boundary layer on a moving ground would experience the same effects, its size relative to the body would require a ground clearance to be an perhaps an order of magnitude less to produce the same effect.

Referring back to the initial questions posed in Section 1, some firm conclusions based on studies described here can be drawn. For the experimentalist, the clear preference would be for a 
moving reference frame study such as that involving a rocket sled, whereby the object may pass over a ground plane with no additional interference - furthermore, at near-sonic speeds, a certain period of "settling time" may be required for the flowfield to fully establish. With sled facilities being expensive and restricted, the symmetry method has been proven to be the most effective in a wind tunnel setting provided blockage is not a significant issue.

Shock-affected lifting body results described in this section provide a useful illustration of the problematic flowfields encountered in particular in the transonic regime - these will now be discussed in more detail with specific reference to their applied context.

\section{High-subsonic and transonic ground effect}

For discussions of ground effect at freestream Mach numbers where flow compressibility is deemed less consequential, the reader is directed to excellent reviews of Wing-in-ground-effect vehicles (for instance, [61,1]), ground effect aerodynamics for race cars [62,63], and high speed rail [64] for extensive discussion of research in these respective fields. For the present purposes, it is merely sufficient to re-iterate that the ground effect for a lifting body serves to enhance lift with little drag penalty due to an increased effective angle of incidence - high pressure under the wing due to a ram effect in the constricted space drives an increased pressure differential around an aerofoil section $[65,22]$. For a highly cambered inverted wing, the ground proximity offers an increased suction due to the venturi-like effect between wing and ground at the lowest point, and is used in many forms of motorsport for downforce.

\subsection{Automotive and rail/maglev}

The front wing of an open-wheel racing car such as a Formula $1 \mathrm{car}$ is responsible for producing as much as $25-30 \%$ of the total downforce, or negative lift, of the vehicle [66], and affects the aerodynamic performance of bodywork downstream. In the last 20 years or so many investigations into inverted wings in ground effect both in wind tunnels and, increasingly, computationally, have been conducted; prior to this there was little available literature directly on the subject despite the ubiquitous use of such devices in motorsport [62]. Researchers studying racing car aerodynamics have identified compressible effects as a likely source of influence on aerodynamic performance in this range, though few studies have been made to date. In Katz's otherwise comprehensive review from 2006 of the aerodynamics of racing cars [63], the single mention of compressibility comes in a brief passage about the Mach 0.85 Blue Flame rocket car.

While shock waves would not form around the external bodywork of a Formula 1 car even in highest-downforce configurations (top vehicle speed and Mach number: $<100 \mathrm{~ms}^{-1}$ and $<M_{\infty}=0.3$, respectively), Keogh et al. explored considerable compressibility effects using RANS CFD $[67,68]$ for a representative realistic vehicle body and concluded that the ground effect predicted markedly different component performance when compared to incompressible results. This had been suspected by Zhang et al. in 2006 [62]. Preceding numerical studies - again using RANS - indicated that a shock wave could form around an inverted wing in ground effect at a freestream Mach number of slightly less than 0.4 for a $3 d$ wing $[69,70]$, and perhaps even less than 0.3 for a $2 d$ section of the same wing [71] were it in a higher-downforce configuration. The geometry used was a simple 1-element front wing with an endplate at a fixed low incidence and (moving) ground clearances of $h / c=0.313-0.067$ for freestream Mach numbers of $0.088-0.4$, and it was expected that a modern open-wheel racing car with a multiple-element wing in a high-downforce configuration would feature even more heightened flow accelerations in the venturi-like channel the wing forms with the ground.

The shock wave was found to increase significantly in strength with reduced ground clearance. Compared to shock-free conditions at higher clearances or lower Mach numbers, the onset of a supersonic pocket at the suction peak of the wing (i.e. the point of closest ground proximity) had a destructive effect on downforce production, caused an increase in drag due to earlier and more significant separation downstream of the shock, and the pitching characteristics of the wing were also affected as a result. For the 2-dimensional section at $M_{\infty}=0.4$, a regular transient shock oscillation formed with an approximate period of $0.005 \mathrm{~s}$, during which $C_{L}$ and $C_{D}$ underwent a standard deviation of over $100 \%$ of the mean value. This would have significant implications for material fatigue from vibration or possible undesirable aeroelastic coupling were the velocity maintained for any significant period of time. Two snapshots of this flow behaviour are reproduced in Fig. 11, showing key points at (a) the point of maximum shock travel and separation, and (b) shock-free flow with the maximum of the separated vortex downstream of the trailing edge (close to the maximum lift and maximum drag condition)

A follow-up study to separate Mach and Reynolds number effects indicated that for freestream Mach numbers above 0.3 it would be preferable to Mach scale than Reynolds scale wind tunnel models for correlation to real-world conditions. This work further highlighted the inability of simple compressibility corrections, such as Prandtl-Glauert [72] or an extension to Göthert's Rule [73], to cope with the non-linear effect on pressure distributions and forces for a highly cambered wing experiencing ground effect. Although the supercritical Mach number for such a wing is above that which an open-wheel racing car could achieve on track, it is well within the realm at which a land speed record or top fuel dragster could operate.

The design of the successful JCB Dieselmax, the vehicle which currently holds the world land speed record for a diesel-powered car (at approximately Mach 0.47), was examined and refined purely with CFD [74]. While the report rightly identifies difficulties in wind tunnel testing for a vehicle up to the Mach 0.5 region, in that such a moving ground does not currently exist and Reynolds number matching is problematic for scale models, the coupled CFD solver used was reported as featuring poor numerical stability. Although the simulations conducted were fully viscous using the Realisable $k-\varepsilon$ turbulence model [75], the mesh featured only unstructured tetrahedral cells at the surface, which would have led to a poor boundary layer representation (as evidenced by anomalous "tiger-stripe" contours on the body surface) and potentially misleading results for skin friction drag. A difference of $8 \%$ in lift and drag was reported between two meshes and, as observed in the report, there was no way to determine which approach was more accurate in the absence of any wind tunnel data. Therefore while parametric comparisons of models may have provided useful information, the reported lift and drag values obtained, on one mesh and with one turbulence model, may well have been discrepant with the actual performance of the vehicle. The designers noted at the time that the flow was predicted to be supersonic at the front wheel region, though no other mention of this phenomenon was made. Given that wheels accelerate flow into the wheel/ground contact patch and produce local $\mathrm{Cp}$ values far in excess of 1 even at low subsonic Mach numbers, the presence of sonic flow in the vicinity as flow accelerates out of this region seems feasible though its effect remains an unknown.

The proposed "American Challenger" rocket-powered land speed record vehicle - one of many designs littering the history 
a
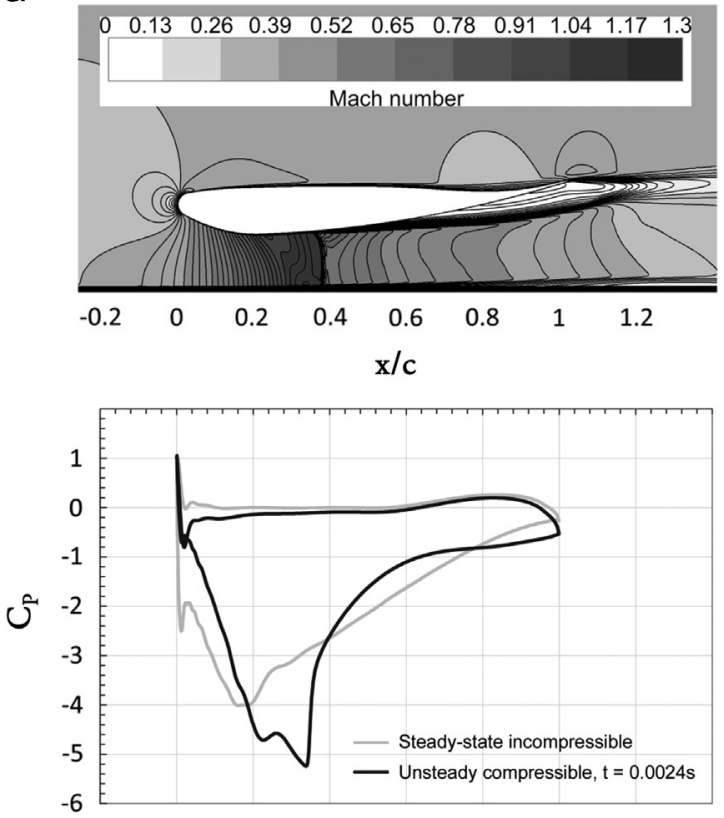

b
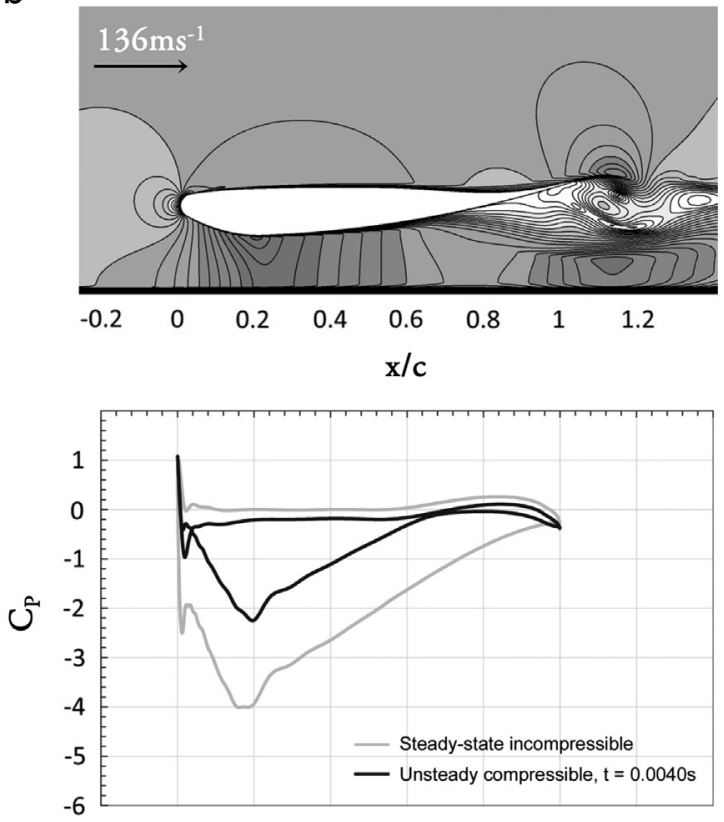

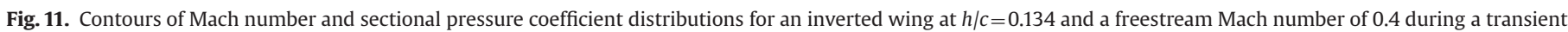
shock oscillation causing boundary layer separation for (a) maximum downforce, and (b) minimum downforce in the regular cycle.

Adapted from [69].

of land speed record racing which were never actually built - was similarly studied using RANS CFD without an accompanying wind tunnel programme. The car was designed to go supersonic, and the initial study [76] looked at a half-model with a symmetry plane. The vehicle featured wing sections in ground effect as struts to connect outlying rear wheels with the cylindrical main body, and bi-convex and diamond aerofoil sections were examined. It was reported that shock-induced force oscillations were significant at Mach 0.8 with the diamond section, and were present but lessened for the bi-convex profile. From Mach 0.6 to 0.8, complete lift force reversal was observed for the full vehicle in certain configurations (negative to positive lift), with an accompanying reversal of the pitching moment sign (positive to negative). Subsequent modifications to the vehicle based on the initial study resulted in a more stable configuration [77] though it was never tested.

As described in the previous section, wind tunnel tests on the Blue Flame rocket car were conducted at Mach numbers from 0.5 to 1.15 using an elevated ground plane [50]. It was noted that shock reflections caused a build up of high pressure beneath the vehicle, leading to a tendency for increased lift as Mach number was incremented. The shape of the vehicle, a long cylinder with an ogive nose and a cockpit and tail at the rear, with outrigger rear wheels, was designed to have a "rounded triangle" cross section to alleviate this pressure build up and promote flow around the vehicle away from the ground vicinity. Originally the outboard rear wheel suspension struts were to be enclosed in a NACA 66 profile swept wing, but wind tunnel tests of these were reported as causing an "adverse influence of wing structure on the pitching moment", and the wake from the front wheels caused interference. Regardless, the driver of the vehicle reported the handling to be excellent with no discernible trend towards lifting or dipping of the car's front during the record-breaking runs; the wind tunnel tests predicted a moderate downforce front and rear but the configuration was changed for the final vehicle design.

A study reported in 2012 [78] revisited the performance of the Blue Flame using RANS modelling to evaluate the forces and moments which likely did occur during the record runs (approx.
Mach 0.84), and also those which may have been experienced had the design been able to pursue its original goal of supersonic speed. A high-fidelity model of the vehicle as-built, based on original line drawings, was subjected to Mach numbers from 0.5 to 1.2 at intervals allowing steady-state simulation using the SpalartAllmaras turbulence model popular for transonic computations [79]. It was reported probable that had the vehicle been able to accelerate all the way to its theoretical top Mach number of 1.2 then the lift force would have significantly exceeded the weight of the vehicle as its fuel load reduced, albeit with a significant nose-down pitching tendency due to the cockpit cabin at the rear of the vehicle on the upper surface. This net lift scenario would have been met close to Mach 1. It is likely that the original wind tunnel tests would not have accurately anticipated this behaviour due to the large ground boundary layer relative to the model, which would have greatly altered the shock reflection behaviour in that region. It is the reflection of the shock waves which raises the pressure on the underside of the forebody and therefore inclines the body to lift even at a slight negative incidence; a misrepresentation of the shock/ground interactions and reflections would likely have resulted in a failure to correctly predict what could have been a catastrophic destabilisation of the vehicle. As it was, the vehicle achieved a successful world record at Mach 0.84 $(1002 \mathrm{~km} / \mathrm{h})$ which stood for 13 years.

That record was broken by Richard Noble's Thrust II in 1983 [80], however a serious thorough aerodynamic development of a land speed record car did not occur again until Noble's successful Thrust SSC programme of the mid-1990s. By then, CFD had evolved into a practical and common tool for aerodynamic design, and inviscid calculations were carried out on simplified models to refine the design [81]. The team insisted on physical testing, however, since at the time CFD was a relatively untested prospect for high speed ground vehicles [51]. Rocket-sled testing was conducted at the Pendine Sands range in the UK as discussed in Section 2.

The Thrust SSC car, being the first jet car on wheels to successfully travel at a supersonic Mach number, was in many respects a working testbed for evaluating the effects of traversing the transonic regime next to the ground. Unfortunately, much of 
the data collected on the car's record runs was lost due to hardware failures, yet striking images exist of the car's bow shock wave ploughing a near-100 $\mathrm{m}$ wide track in the desert floor and the shock waves produced by the vehicle's upper surface at nearsonic speeds. These shocks are canted at an angle which resulted from the local flow velocities equating to well over Mach 1. Due to the relatively rigorous testing described in the previous section, the car was not at risk of becoming airborne. However, two unexpected phenomena occurred - first, as the car accelerated through the Mach 0.8 region a significant sudden "jump" leftwards (up to $100 \mathrm{~m}$ ), requiring full lock from the driver to correct, was experienced after which the side force lessened and the car became stable again. A CFD study conducted a decade later [82] indicated that the likely cause of this was a shock wave forming ahead of the rear wheels, which were offset (staggered) rather than tandem in configuration; the subsonic flow behind this shock threading through between the staggered wheels produced a large side force, exacerbated by the flow asymmetry affecting other parts of the bodywork. Since all the original CFD of 1993 as well as the rocket sled testing utilised a symmetrical model (i.e. wheels side-by-side instead of the staggered-wheel version of the final vehicle), this flow effect was not anticipated.

The second unexpected phenomenon was described by the driver and engineers reporting the car sliding at its top speed, suspected to be a result of the quasi-fluidisation of the desert surface caused by the shock wave pulverising the compacted earth ahead of the wheels. This latter phenomenon is yet to be investigated experimentally or numerically. An interesting similarity to this fluidisation of the desert surface stems from the contact phenomena of sled testing at hypervelocities, where the solid-solid contact of sled/rail interaction causes the melting of the surface leading to a fluid interface, the liquid film preventing further physical contact and causing potential local vaporisation [83]. This affected the shape and nature of steel track gouging patterns observed. However, since that is primarily a solid-solid interface scenario leading to a fluid-structural problem, the analogy will not be further explored here.

Remaki et al. [84] simulated the enhanced drag for the Bloodhound SSC car - under construction at the time of writing - using a multi-phase gas-particle model to account for sand rising due to shock/ground interaction. They concluded that the drag augmentation was not likely to exceed $10 \%$ as a result of sand particles impacting the car at high velocity. The model can be considered a first significant insight into this effect, but does not fully describe the nature of the shock/ground interaction in terms of the semiliquefied effect described by those involved with Thrust SSC [51].

Indeed, wheels and their interaction with the ground at transonic and supersonic speeds has been a particular focus of the Bloodhound SSC vehicle designers. With many of the same team from the Thrust SSC effort, and a confirmed faith in CFD to produce accurate results, a study reported the application of highfidelity RANS modelling using the Spalart-Allmaras turbulence model to choose a wheel shape and evaluate a preferable spacing in a parametric study [85]. A parallel arrangement was chosen over a staggered one, for the sake of stability over a narrow range of steering and yaw angles. Mach number contour plots indicate that the ground constraint coupled with the low clearance of the main vehicle result in considerable jet flow between the narrowly spaced front wheels, with a bow wave sitting ahead of the wheels and flow re-accelerating to a supersonic level through the gap. This would have occurred on the Blue Flame vehicle as well but could not be studied at that time.

Viscous RANS full car modelling of the "Bloodhound" was conducted using meshes of the order of 35 million cells with a hybrid approach, and the numerical implementation of both a moving ground and rotating wheels [86]. A significant design challenge reported was the elimination of lift at the rear of the vehicle due to fairing design for linking the rear wheels to the fuselage. The CFD permitted detailed analysis indicating the source of the lift was the strong shock generated by the rear wheels and suspension structure and the interaction of this shock system with the main vehicle body. Considerable downforce in this region was predicted at $M_{\infty}=0.5$, compared to the lift force observed at the design speed of Mach 1.3. The simulations led, through a process guided by a design of experiments approach, to a redesign of the region to avoid this effect.

Other ground-based, person-carrying high speed transportation is not subject to such extreme conditions, although with conventional rail vehicles pushing into the Mach 0.3 range and experimental Maglev transit systems likely to reach Mach 0.5 in the near future, some supersonic flow interactions are likely to become a focus of more research in this area [87].

The strong compression waves formed when modern high speed $\left(M_{\infty}<0.3\right)$ trains enter tunnels are relatively well-studied [88], but they do not in themselves constitute a similarity with ground effect unless we consider the local acceleration of flow around the curved upper surface of a train when in close proximity to a tunnel roof. The "piston effect" produces upstream compression waves which coalesce to form a discontinuity and an undesirable acoustic issue analogous to the sonic boom from a supersonic aircraft, but the weak shock waves travel ahead of the body and are not necessarily of significance to the stability or general aerodynamics of the train while in the tunnel [89].

A study by Baron et al. into compression wave effects based on different tunnel blockage ratios indicated that for a very high blockage ratio, with a train Mach number of 0.35 and after $300 \mathrm{~s}$ of time in an exceptionally long tunnel, the flow at the rear of the train had become locally sonic forcing a strong recompression wave at the tail of the carriage [90]. The analogy was made to flow in a de Laval nozzle. Mach numbers as high as 1.7 were attained and varied strongly with tunnel diameter, train length, distance from the tunnel exit, and time in the tunnel. Resultant increases in power required to maintain train velocity due to increased drag were not discussed, though from the projectile research described in the present paper we may assume that the drag increase could be significant if shock-induced separation or significant wake disruption occurs due to the shock behaviour.

As a close relationship exists between the internal tube pressure and the blockage ratio for aerodynamic drag [91], a conventional train travelling through air at $700 \mathrm{~km} / \mathrm{h}$ was simulated by Kim and Kwong using RANS CFD for a variety of ratios to show that a significant shock reflection pattern can form in the wake and indeed interfere with the train's normal (free air) wake as well as instigate vibrations in the tunnel wall. The critical Mach number in that instance was found to be independent of tube pressure and thus dependent on blockage ratio entirely.

Hammitt [92] first noted this propensity for tube vehicles to produce choking and sonic conditions in 1972, reasoning that a long train in a long tunnel will produce the most severe effects due to boundary layer build-up and lack of alleviating conditions near the train from a tunnel exit. The predicted shock reflections from the walls at the rear of the vehicle led to a conclusion that a propulsion system for such a vehicle ought to be located at the fore of the body rather than the tail.

However, when looked at on the macro scale, this can be considered to be exclusively "tube" or tunnel flow rather than ground effect flow, a separate area for which a wealth of literature exists. We can surmise for the case of a high speed train that changes to lift and stability in a tunnel are due to the asymmetry of the train shape rather than a specific ground interaction leading to the possible wave formations around the vehicle.

In contrast to rail-based transportation, aerodynamic instabilities would be difficult if not unacceptable for a fast air cushion or 
maglev transportation vehicle, as variations in the ground clearance affect the position of the vehicle within the magnetic field for the latter case, and therefore the levitation force imparted to the vehicle [93]. For this reason it would be highly preferable to avoid the onset of transonic flow features which may trigger sudden changes in stability.

\subsection{Aircraft and lifting bodies}

Very little real-world flight data exists on supersonic flight in close ground proximity. A now-declassified report [98] on the lowaltitude overflight of supersonic aircraft - namely an F-104 Starfighter and a Republic F-105D Thunderchief (not explicitly named in the report but easily discernible in hindsight) - appears to include the first documented methodical measurements of the pressure signal of supersonic aircraft in ground effect. The tests were primarily concerned with shock wave potential to damage structures and break windows, etc., but provide almost certainly the first comprehensive measurements of strong shock/ground interaction from a low-altitude supersonic overflight.

The study also highlights the differences in pressure signals obtained from each aircraft. In contrast to the nature of the "sonic boom", the low-altitude pressure signal constitutes discernible waves from each aspect of the aircraft's geometry, and thus the difference in ground pressure (shock strength), lateral effects, and local shock angles have a marked influence on the measurements. Overall trends between aircraft types were the same, however.

As the results are comparable, data is presented here for the F-105 as its larger wing span (34.5 ft) and mean wing chord ( $8.6 \mathrm{ft})$ would have made it more susceptible to the ground effect for an equivalent altitude. At the lowest tested ground clearance, $h / l$ for the aircraft and $h / c$ for the wing sections alone would have been 1.28 and 5.8 respectively. Altitudes from $50 \mathrm{ft}$ to $890 \mathrm{ft}$ were reported. The charts presented in Figs. 12 and 13 have been adapted from the original data to show metric values and additional data, and non-dimensionalisation was implemented where possible. Measurements at several metres above the ground, as well as on the ground plane, are presented to highlight the increased overpressure at the ground and the distinctive pressure signals produced. Results agreed well with generalised near-field shock theory based on the Mach number, indicating that the level of ground clearance was not close enough to produce any discernible unexpected effects of a magnitude outwith the (considerable) margin of error in altitude and aircraft location relative to the transducers with successive flights.

It is noted that the test pilots involved in these tests did not report "any unusual operational problems" in accomplishing the

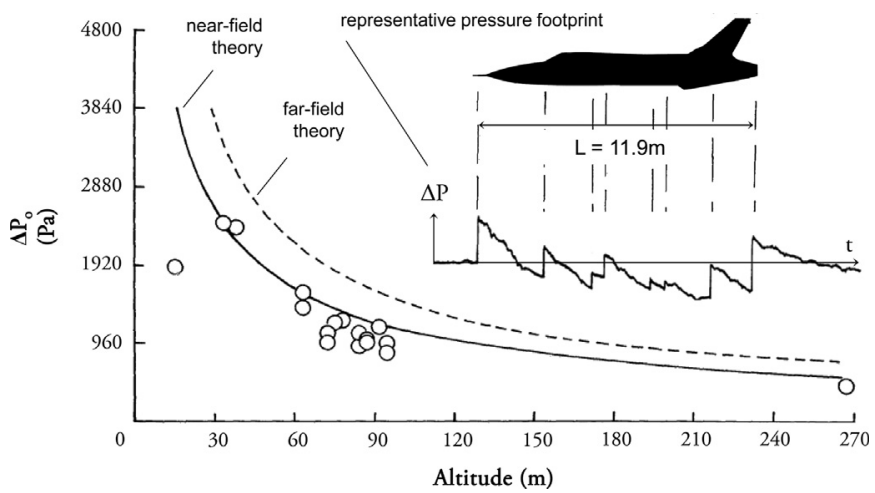

Fig. 12. Peak total pressure at a measurement station above the ground vs. altitude (ground clearance) for a military jet overflight at approximately Mach 1.1, and inset: sample pressure history of a typical overflight.

Data from [98].

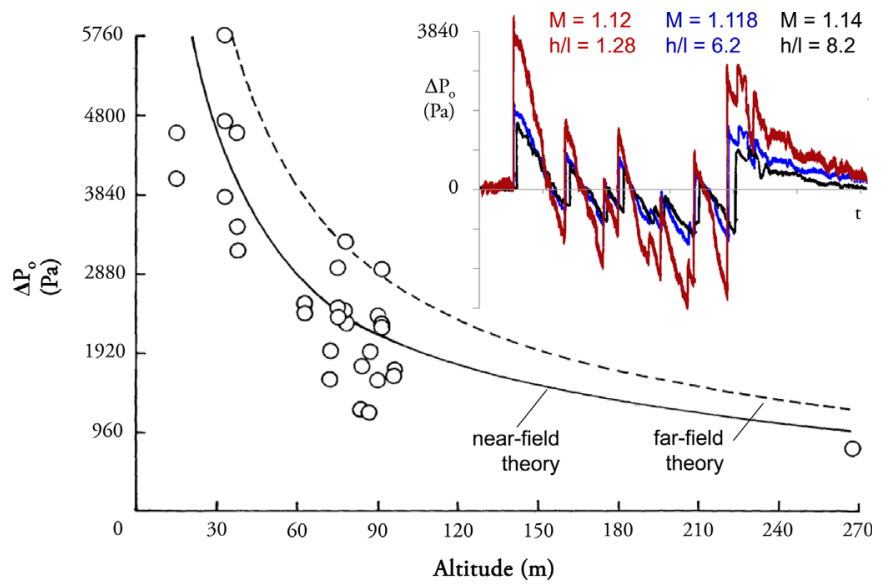

Fig. 13. Peak total pressure at a measurement station on the ground vs. altitude (ground clearance) for a military jet overflight at approximately Mach 1.1, and inset: sample pressure history of a typical overflight for different Mach numbers and ground clearances.

Data from [98].

flights, and no appreciable turbulence was encountered, nor any "unusual control problems", indicating a relatively smooth, quasisteady-state when test altitudes were obtained (during early morning hours to reduce rising thermal effects and increased wind/turbulence). Shock wave angles were measured from the multiple-microphone measurement arrangements and it was concluded that in no test did the reflected shock waves from the ground impinge on any parts of the test airplanes. As shown in the following section with reference to projectiles, this may have been an over-simplifying assumption as it fails to account for the adjustment of the shock reflection state at the ground boundary and therefore the wave reflection angle, and is instead based on a simple regular reflection at the appropriate angle for shocks for the given Mach number and nose-cone angle.

Similar studies followed in 1968, as reported by Nixon et al. for the low-altitude overflight of an F4-C Phantom II [99]. Near-identical effects were noted apart from an increased pressure differential that could be attributed to the slightly higher Mach number (approx. 1.2) and the different body shape of the Phantom as it made passes at an $h / l$ of approximately 1.55 and above. The conclusions also noted that low-altitude shock/ground close-proximity sonic booms do not "harm people directly" and livestock appeared to be unbothered, though small animals may experience injury, distress or death from cardiacarrest from near-field shocks and sonic booms measured from aircraft at much higher altitudes [100]. As indicated in Fig. 14, the pressure rise lateral to the overflight path drops off markedly as the waves diffuse either side of the aircraft, with the strong shock (and therefore any likely reflection) largely confined to a narrow region - this may explain in part the considerable variability in the results of Fig. 13, given that proximity to the pressure probes was far from perfectly repeatable with each overflight.

Analytical studies have been made using boundary element methods which consider subsonic and supersonic compressible flow over aerofoils in ground effect [96,97], but the formulation can only be generally applied to thin aerofoils. Despite this, results were presented for a (relatively thick) NACA 4412 aerofoil at $0^{\circ}$ incidence, indicating that for a freestream Mach number of 0.5 , the compressible case predicts $C_{L}$ as being $12 \%$ higher for ground clearances greater than a height-to-chord ratio $(h / c)$ of 0.5 , below which the compressible $C_{L}$ increasingly agrees with the incompressible prediction. No significant discussion was offered as to why this occurs and the method does not facilitate a detailed examination of a flowfield.

Doig et al. [101] presented a parametric numerical study, as validated extensively against the RAE2822 section transonic wind 

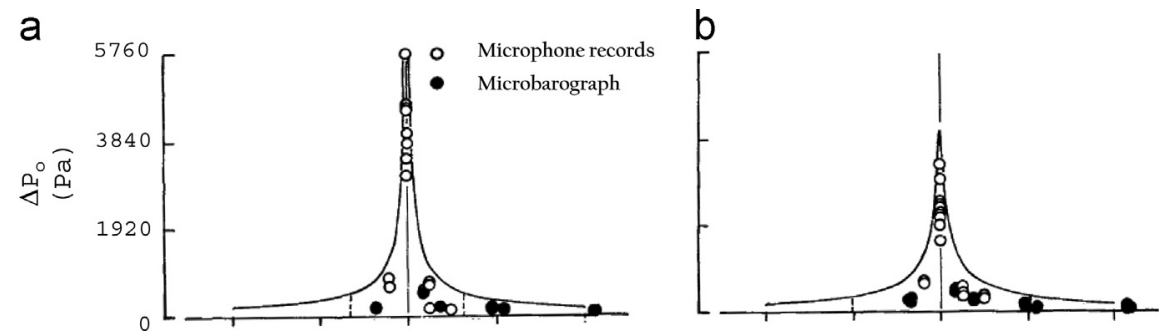

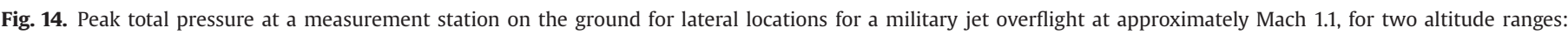
(a) altitude range, 50-125 ft and (b) altitude range, 210-260 ft.

Data from [98].

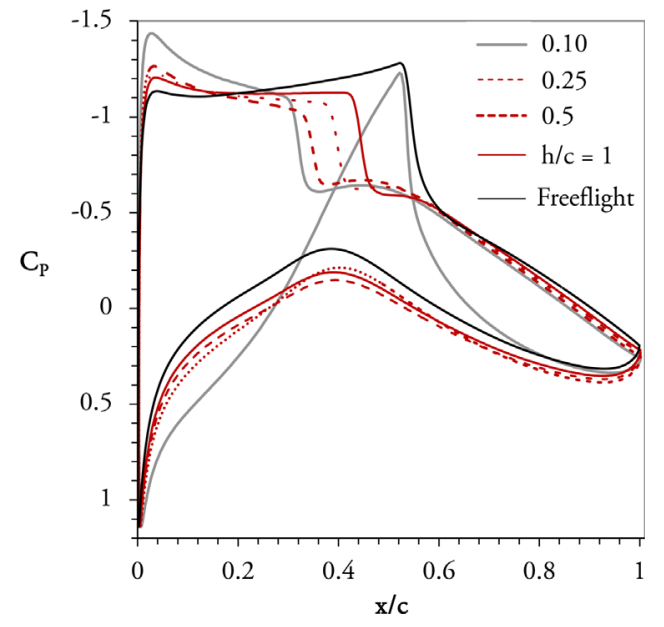

Fig. 15. Sectional pressure coefficient distributions around an RAE2822 for different ground clearances and a freestream Mach number of $0.73, \alpha=2.8^{\circ}$.

tunnel experiments described in the preceding section, of a parametric variation of ground clearance ( $h / c$ from 2 to 0.1 ), angle of incidence $\left(0-6^{\circ}\right)$, and Mach number (0.5-0.9). Again they used RANS modelling, transient in some cases where the flowfield would not settle to a steady-state, with fully structured meshes and a modified variant of the 1-equation Spalart-Allmaras turbulence model. The model appeared to be effective in the absence of any significant boundary layer separation. Although the aerofoil section was not designed to operate efficiently at the high end of that Mach number range, and is not of the flat-bottomed type commonly investigated for ground effect problems, it was presented as a useful comparison to freeflight results which have been used extensively for code validation. It was also reasoned that higher angles of incidence on the wing would effectively take an aircraft out of ground effect in a practical flight situation, and thus were neglected.

As shown in Fig. 15, decreasing the ground clearance, for a typical incidence of $2.79^{\circ}$ and a fixed Mach number of 0.73 , yields significant changes in the sectional pressure coefficient.

The upper surface shock location moves progressively upstream from its freestream location, by about $25 \%$ of the chord by $h / c=0 \cdot 1$. It also gradually reduces in intensity, resulting in a less severe pressure increase across the wave. One of the main reasons for this behaviour is the downward movement of the stagnation point at the leading edge, which also increases the strength of the suction peak near the leading edge on the upper surface. This increase in the effective angle of incidence draws the shock upstream, and creates a stronger adverse pressure gradient across the forward portion of the upper surface leading to the earlier, weaker shock and a reduction in the region of 'rooftop' pressure distribution. The flow remains attached at the foot of the shock.

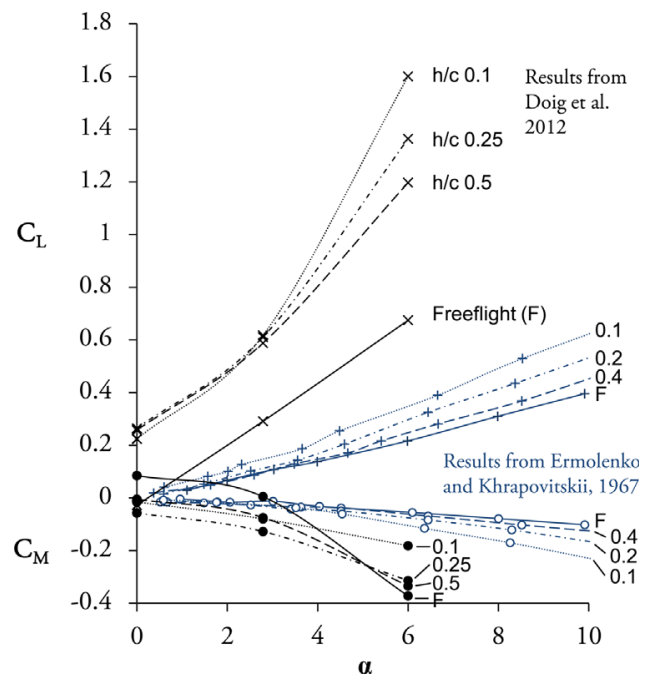

Fig. 16. Lift and pitching moment coefficients vs. angle of incidence for aerofoils at various ground clearances and a freestream Mach number of 0.9. Data sources: $[9,102]$.

At the same time, the pressure distribution on the lower surface of the aerofoil is affected as the air which is forced underneath is increasingly constricted by reducing ground clearance. As more mass is directed over the upper surface, the lower velocity under the aerofoil causes higher pressure in the region between the aerofoil and the ground, particularly near the leading edge as the stagnation point is drawn downwards, and thus the maximum pressure difference between the upper and lower surface is exaggerated with increasing proximity to the ground.

These general trends hold until the lowest ground clearance, $h / c=0.1$. In this case, the flow between the aerofoil and the ground has accelerated to supersonic local Mach numbers, causing a strong shock wave at approximately $x / c=0.54$. The normal shock is stable and of greater strength than that of the upper surface. As a consequence of all this, lift increases significantly with decreasing ground clearance, as expected, but with a $25 \%$ drop between $h / c=0.25$ and 0.1 due to the formation of the lower shock, which also produces an $80 \%$ drag rise.

In the remainder of the parametric study the relationship between the primary variables was explored and the resultant aerodynamic coefficients were found to be sensitive to all - a change in angle, Mach number or ground clearance could result in the formation or disappearance of upper or lower shocks at the lower end of the Mach range, and at the higher range more predictable trends were observed but there was reduced efficiency.

Lift and pitching moment results are presented for the section in Fig. 16, for Mach 0.9 to facilitate comparison with the data of Ermolenko and Khrapovitskii from 1968 [102], where analytical results for a nominally thin section hinted at promising performance (the cambered 2822 section producing much higher lift). 


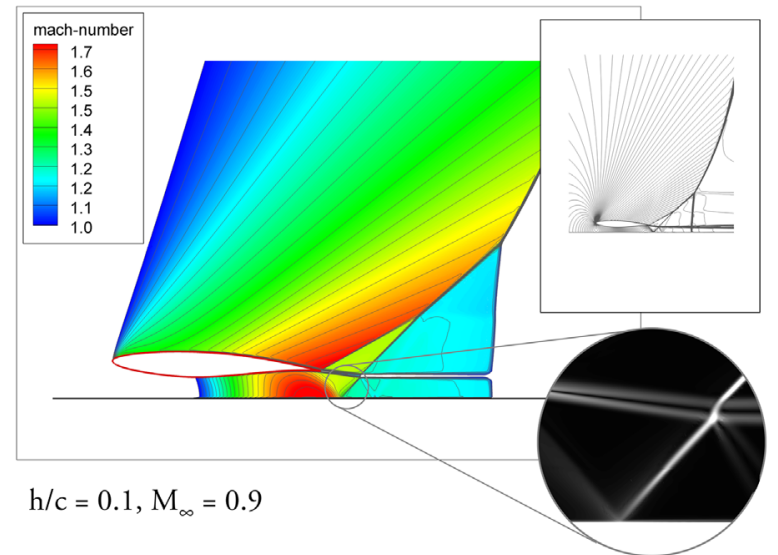

Fig. 17. Contours of Mach number and numerical schlieren of the shock reflection pattern formed by an RAE2822 aerofoil section at $h / c=0.1$ and freestream Mach number 0.9 .

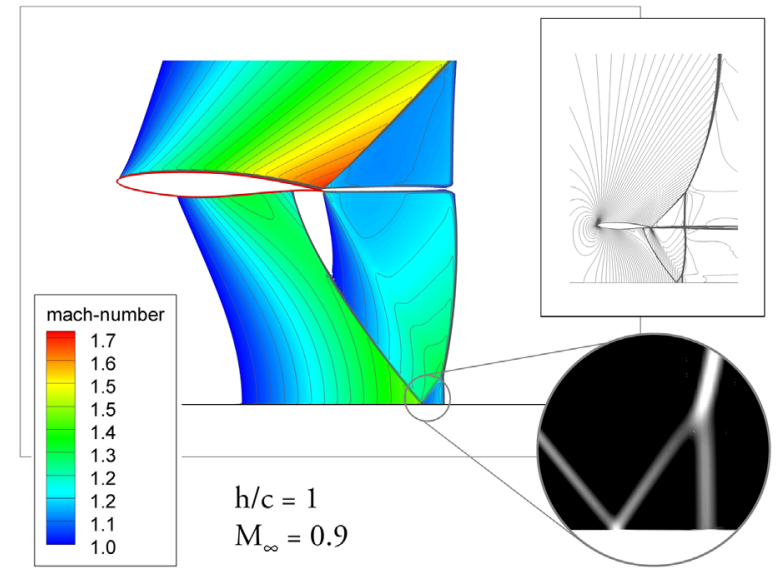

Fig. 18. Contours of Mach number and numerical schlieren of the shock reflection pattern formed by an RAE2822 aerofoil section at $h / c=1$ and freestream Mach number 0.9 .

The predicted flowfield is complex, as indicated in Figs. 17 and 18 for $M a c h=0.9$ and ground clearances of $h / c=1$ and 0.1 . At the former clearance, the upper surface shock sits at the trailing edge while the lower surface shock is generated by the change from convex to concave upstream of that point. This shock reflects regularly from the ground plane (reminiscent of the shocks seen in Fig. 2), but forms a 3 shock structure immediately downstream to turn the flow back to parallel, the upper portion of this secondary main shock interacting with the wake and forming a large-scale structure with that of the upper surface shock.

At $h / c=0.1$, the overall peak local Mach number occurs at the ground plane immediately under the trailing edge, and there is only a weak coalescence of waves reflecting from the ground - the terminating shock sits close to 1 chord length downstream. The onset of shock waves in the flowfield has a disruptive effect on performance, particularly at the lowest clearances, where the early formation of a shock wave between the aerofoil and ground can lead to a sudden drop in the production of lift and an accompanying early transonic drag rise for the section. It can also lead to the development of unsteady shock oscillations on the lower surface, and has a considerable effect on the pitching moment of the section, lending it a nose-up moment at low clearances where it would normally have a nose-down moment at higher clearances and in freeflight.
It has been shown that modelling wind tunnel floors and ceilings can alter the predicted fluctuation levels of a shock wave on a reproduction of a nominally 2-dimensional lifting section experiment [103], and therefore the wind tunnel wall interference similarity to ground effect flows may again provide insight into the specific ground influence. Without dedicated experiments that can provide accurate frequency information on shock passage in a buffeting scenario, it remains too early to comment decisively on whether the ground specifically invokes an earlier buffet boundary; unsteady RANS itself is a questionable means by which to investigate such phenomena.

The aerofoil ground effects described thus far serve to affect the drag considerably, thus while the lift results confirm Ermolenko and Khrapovitskii's hypotheses about improved lift well into the near-sonic bracket, the aerodynamic efficiency of the wing is affected detrimentally as soon as the supercritical Mach number is exceeded. Furthermore, the summary in Fig. 19 shows that flight in close ground effect is no longer an enhancement overall as it is for freestream Mach numbers below 0.7, or 0.6 for $h / c=0.1$ where a shock has already formed between the aerofoil and the ground.

The fact that ground effect produces a higher $L / D$ at a higher angle of incidence $\left(6^{\circ}\right)$, where a shock doesn't form on the lower surface ahead of the trailing edge, shows promise that a bettersuited, more carefully contoured aerofoil section may prove effective into the near-sonic region. It is worth noting that while

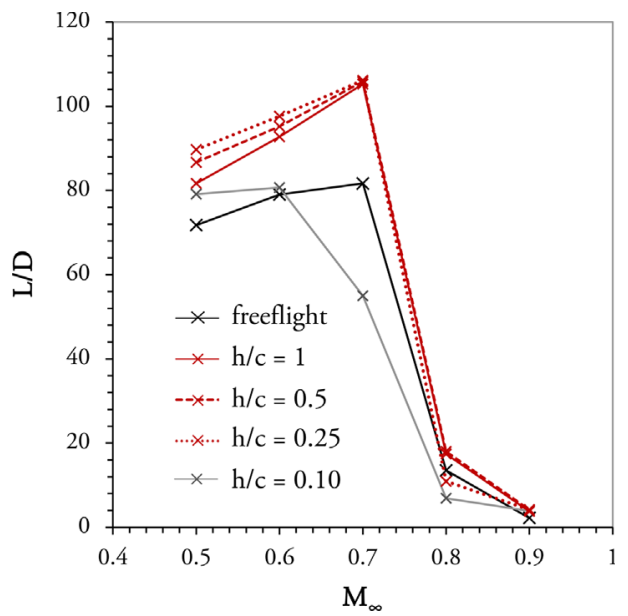

Fig. 19. Aerodynamic efficiency of an RAE2822 aerofoil in ground effect at $2.8^{\circ}$ incidence with increasing Mach number.

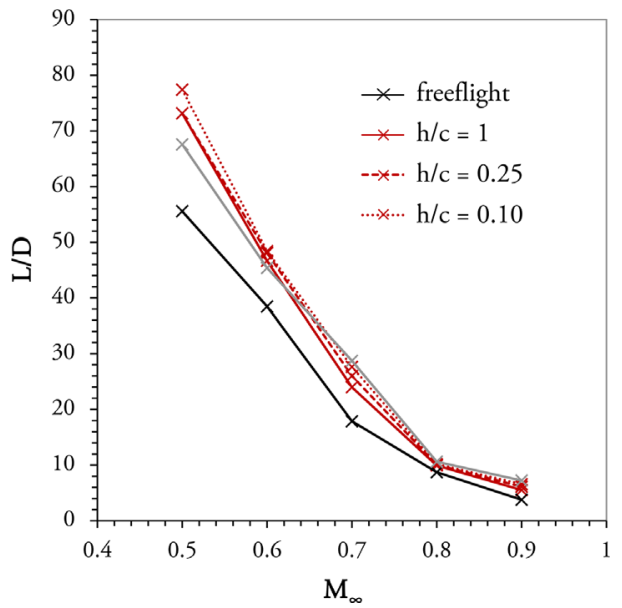

Fig. 20. Aerodynamic efficiency of an RAE2822 aerofoil in ground effect at $6^{\circ}$ incidence with increasing Mach number. 
the $2.8^{\circ}$ case offered continued rise of the $L / D$ ratio up to Mach 0.8 for all clearances but $h / c=0.1$, at $6^{\circ}$ the $L / D$ ratio declines steeply with increasing Mach number as a result of the forward movement of the upper surface shock as the stagnation point is drawn downwards, reducing wave drag but also depleting the range over which the "rooftop" low pressure region acts.

These general trends were also observed in a subsequent CFD study involving the swept, tapered, symmetrical ONERA M6 wing [104], following the same numerical procedure extrapolated to a 3-dimensional scenario. The effects of decreasing ground clearance on the wing tip vortex were pronounced, as seen in Fig. 22; in subsonic ground effect for a lifting wing, the vortices tend to travel outboard of the wing [105] - more so with decreased clearance or increased wing loading. For the swept wing at $h / c=0.1$ the vortex also travelled outboard for a representative flight case of Mach 0.84 and incidence of $3^{\circ}$. However, this was found to be a result of force reversal in the tip region due to disruptive shock-separation (tip stall) of the flow from the leading edge, whereas the rest of the wing inboard experienced increasing suction due to a forward movement of the primary upper surface shock and decrease in its strength. Therefore as the wave drag from the main shock decreased, it was overtaken by induced drag from the greatly increased tip vortex. The vortex still experienced a significant upwards trajectory in all cases, as is characteristic of ground effect flows.

Doig et al. concluded that transonic flight at low-level would prove challenging and in terms of cruise performance would require careful tailoring of wing section characteristics to avoid any destabilising lower-surface shock suddenly forming [94,95,101]. Without exception, the transonic drag rise was more pronounced, and occurred at an earlier Mach number, than for the freeflight wing.

It has also been established that at subsonic Mach numbers for WIG vehicles built and tested, the natural stability of such a vehicle in roll is excellent, since the descent of one wing increases the lift due to ground effect, providing a natural restoring moment [7]. Were the descending wing to be close to its lower-surface critical Mach number, this effect could be dangerously reversed.

Rhosdestvensky outlines that the influence of compressibility in ground effect is more enhanced than in freeflight, and indicates a larger increment of lift for wings of moderate and large aspect ratio in extreme ground effect [1]. Noting the possibility for formation of shock waves at high subsonic Mach numbers, an attempt was made to apply the Glauert correction factor to a flatbottomed wing to account for compressible effects using modified linear as well as so-called "extreme ground effect theory" [106].
This theory predicts a relatively monotonous decrease of efficiency with increasing Mach number, though the limitations of the method imply that it is a flat section with no discontinuities (i.e. shock waves). Fig. 20, 21 presents a comparison of the results predicted from this method compared to those obtained by Doig et al. for the swept, tapered symmetrical section at an incidence of $3^{\circ}$ [104], showing agreement in terms of general trends and, approximately, values involved - however the latter RANS results highlight the effects of lower-surface shock formation at $h / c=0.1$, causing immediate and debilitating loss of aerodynamic efficiency. The results also echo the findings presented for a 2-dimensional section in that aerodynamic efficiency is not uniformly improved at all ground clearances, and that simplistic compressible corrections are not able to capture the shock-dominated flowfields that are clearly common at high subsonic Mach numbers.

The high-resolution, albeit time-consuming CFD results, point to the onset and behaviour of shock waves to be dependent on ground clearance, freestream Mach number, but also local camber and curvature of the wing or any geometry in question. Accurate prediction of the flowfields is therefore likely to escape the existing analytical treatments for compressible ground effect such as those proposed by Dragos et al. [96,97] where a direct boundary integral equations method was applied to subsonic flow with circulation past thin aerofoils in ground effect. However in the simpler, more predictable supersonic regime, more acceptable results may be gained from such methods.

For magnetically levitated take-off assist for aircraft or launch assist for lifting-body re-usable spacecraft, only a finite time will be spent in the transonic regime and in ground effect, however that short period may be a crucial one and the most challenging aerodynamically. The ability to accelerate a launch vehicle to transonic Mach numbers (i.e. in the vicinity of the transonic drag rise) over a relatively short, inclined (i.e. mountain) track before conventional rocket propulsion is initiated could greatly reduce the vehicle mass due to reduced fuel requirements [107]. While organisations such as NASA consider the idea and technology to be viable [108], no active investment in developing and testing such systems is occurring at the time of writing.

The Maglifter concept, which gained some traction in the mid1990s [109], was based around the notion of accelerating a shuttlelike vehicle up to near-sonic Mach numbers over an inclined, enclosed tunnel of several miles in length, implying that the winged spacecraft would operate in transonic ground effect for a considerable time before reaching its launch altitude.

A similar concept explored by Yang et al. [110] in the intervening years noted that the lift, drag and moment characteristics of a

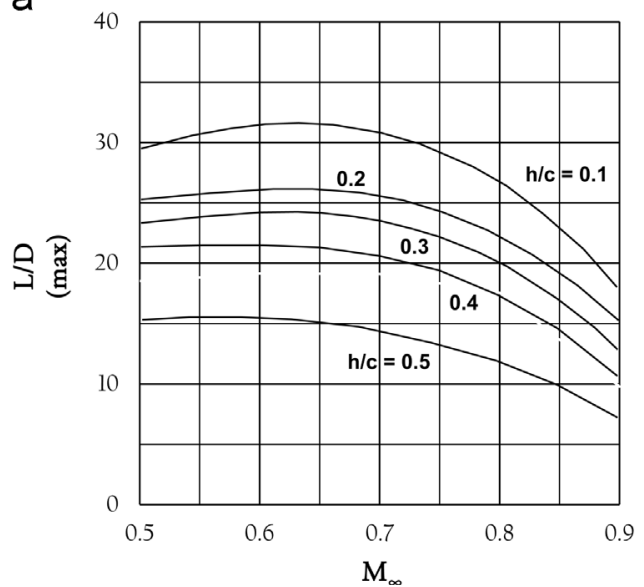

b

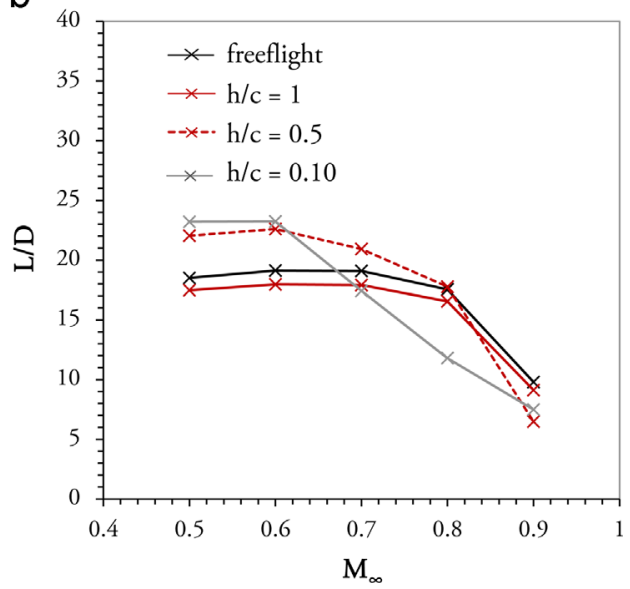

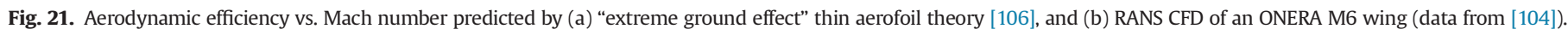


a rail-mounted winged spacecraft were predictable and favourable, yet neither the Reynolds number and Mach number nor the ground representation technique for the $1 / 59$ th model are quoted despite the study being aimed at a $1000 \mathrm{~km} / \mathrm{h}$ launch speed: such results must be treated with caution.

Tomita et al. [111] have even proposed the design of a Mach 0.5 cruising catamaran Ekranoplane with a large wing/deck central section from which a re-usable space access vehicle could be launched, however it would be likely that the launch vehicle would be out of ground effect almost immediately due to a launch angle of $15^{\circ}$ and rapid acceleration away from the host.

The studies outlined here indicate that the transonic drag rise for such a launch in ground effect is likely to occur at an earlier velocity than would be the case for a vehicle in free air, and a more prolonged period spent in the buffet region would require testing from a structural standpoint. These results present an interesting contrast with low-speed ground effect where a lifting body will typically experience enhanced lift due to an increased effective angle of attack - with the formation of a lower surface shock the pressure can be raised suddenly and more dramatically, causing lift augmentation if the high pressure region acts over enough of

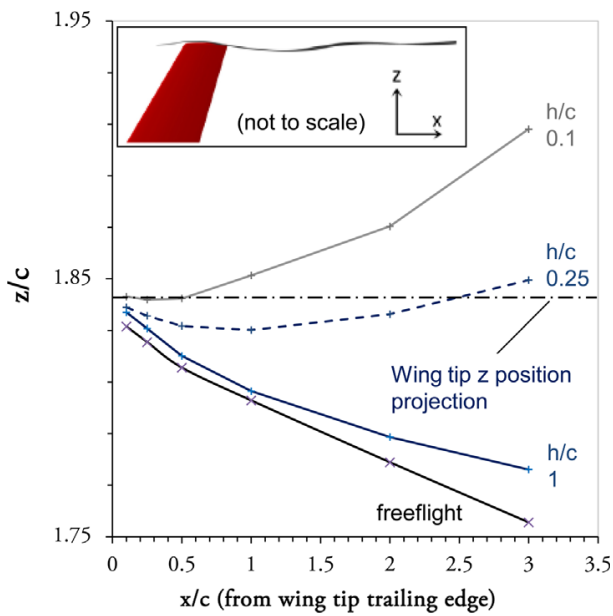

Fig. 22. Tip vortex core lateral $(z)$ location downstream of an ONERA M6 wing at various ground clearances. the surface, however a mixture of downforce and lift that is highly dependent on camber and geometry as well as incidence and ground clearance is possible for any slight change in configuration. At fully supersonic speeds, increased lift is essentially ensured, however efficiency is almost certain to suffer as considerable drag rises associated with shock/BL interactions and wave drag are present and do not allow any perceived "benefit" to being in ground effect.

\subsection{Projectiles}

Flowfields similar to those previously observed for land speed record vehicles (such as the Blue Flame in particular) have been investigated for projectiles in close ground proximity in a Mach number range of 1.1-1.2. The use of live-range tests, wind tunnel experiments, and CFD (as described in Section 2) led to a relatively detailed examination of the effect of shock reflections and ground interactions and their effects on the aerodynamic characteristics of the projectile.

The initial experiments described by Kleine et al. [55] for a zero-incidence projectile at Mach 1.1-1.2 indicated that the presence of the ground affected the shock shape of the detached bow shock, which, as ground clearance was reduced, was observed to bend forward or eventually straighten and move upstream of the projectile. This avoided the establishment of the reflected shock seen at higher clearances of several diameters. Fig. 23(a) shows schlieren images extracted from the original live-range tests which show the flowfield adjusting as the projectile travels over the leading edge of the ground plane. Wind tunnel results, using the symmetry method, indicated the likelihood of a normal force not seen at higher clearances and in freeflight, but the effect of this was reasoned to be "relatively minor". Repeatability in the wind tunnel was obviously better than for the live range experiments where slight variations in ground clearance and projectile angle of incidence were present from test to test; however, to better quantify the aerodynamic characteristics of the projectile and the surrounding flowfields, two numerical studies followed the initial experiments.

The first, by Carriage et al. - using RANS in Fluent (with the $k-\omega$ SST turbulence model [112]) and a non-spinning, symmetric projectile - identified three distinct conditions dependent on
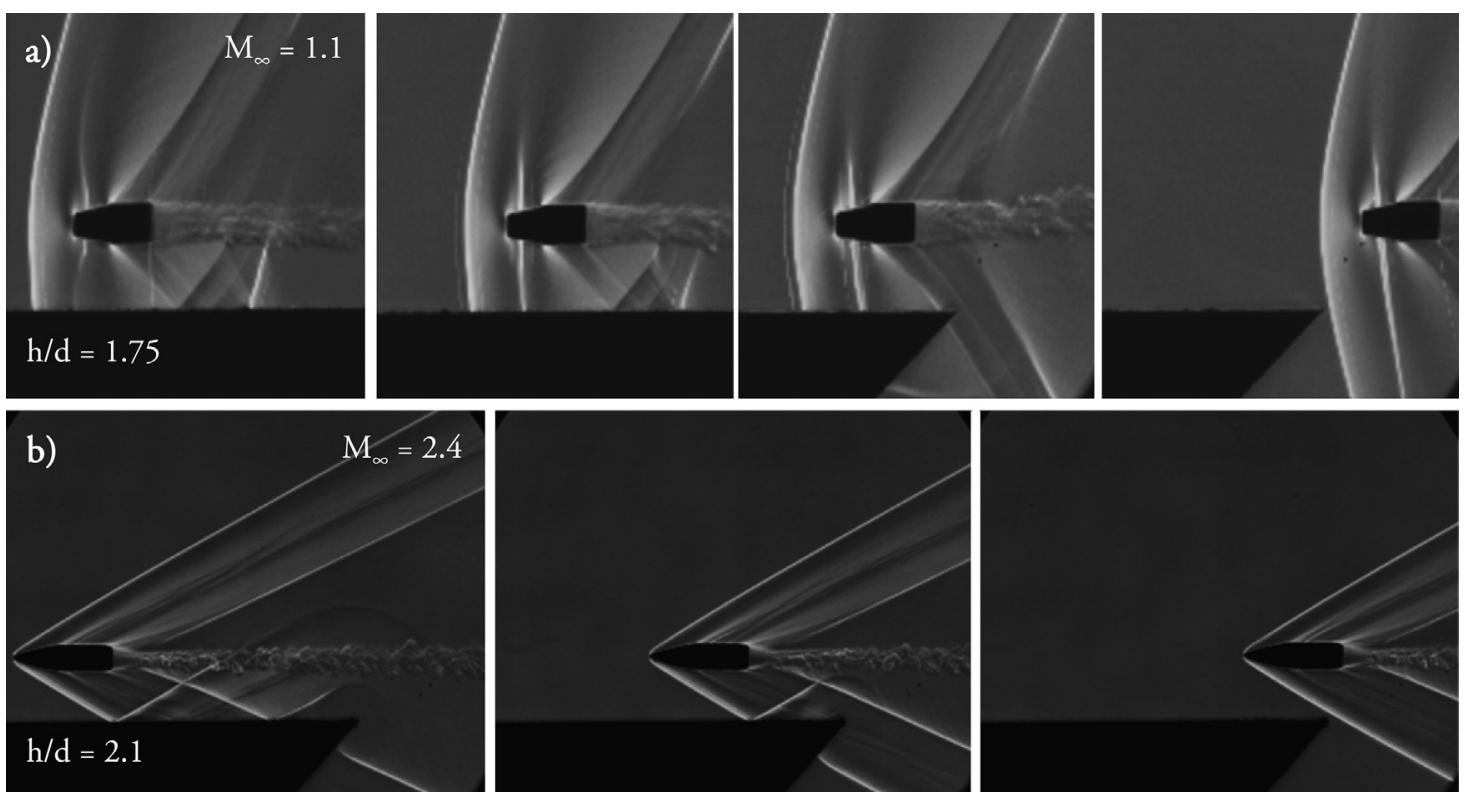

Fig. 23. (a) Blunt-tipped projectile at $h / d=1.75$ at Mach 1.2 and (b) standard NATO $5.56 \mathrm{~mm}$ projectile at Mach 2.4 and $h / c=2.1$. 

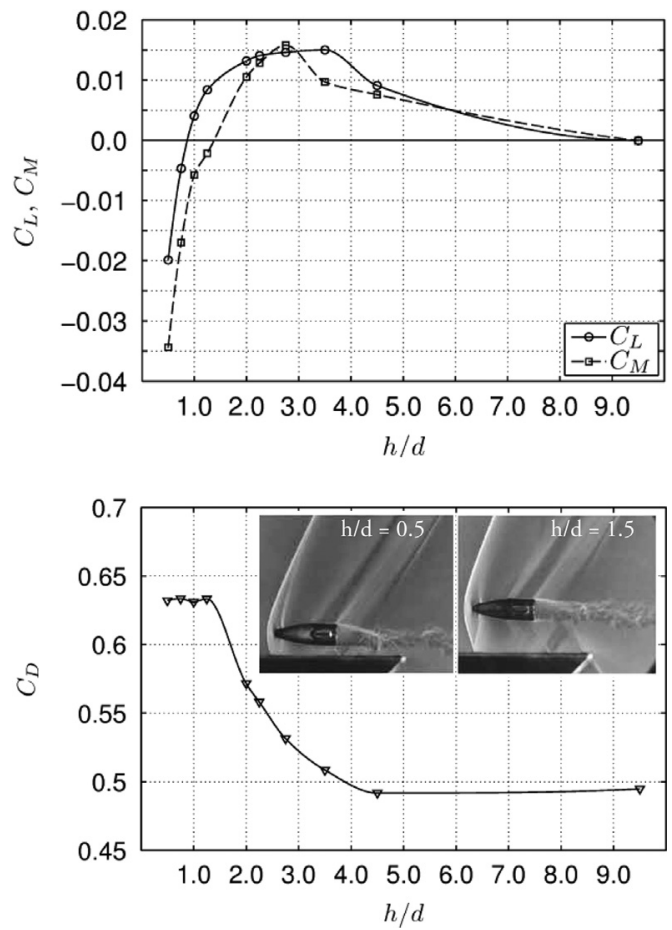

Fig. 24. "Lift" (normal), drag and pitching moment coefficients vs. ground clearance for a projectile at Mach 1.2.

Adapted from [43].

projectile ground clearance, based mainly on a criteria of how the forces and moments were affected [43]. Condition A was characterised by a normal force in the direction of the ground plane, accompanied by a nose-up pitching moment, and was observed at $0.5<h / d<0.85$. Condition B was deemed to occur between $0.85<h / d<1.5$, notable for a normal force acting away from the ground plane, with a minor nose-up pitching moment. This force reversal in the vicinity of the ground plane is similar to those noted for rocket sleds and the aforementioned land speed record cars, further confirming that it is a general characteristic of nonlifting bodies as shocks form underneath, though the Mach number and ground clearance at which this will happen will be strongly dependent on the object's geometry.

For the projectile, a third state, labelled Condition C, was observed at $h / d>1.5$, in which a mild normal force acting away from the ground plane and a small nose-up pitching moment, with peaks in both interpolated to occur at close to $h / d=3$. As in the experimental program, the shock stand-off distance was noted to increase rapidly with a reduction in ground clearance. The force and moment trends are reproduced in Fig. 24, appended with images of the flowfields originally seen in the live-range tests.

A more detailed numerical investigation, also using RANS with the $k-\omega$ SST turbulence model (albeit with meshes an order of magnitude finer, and a spinning projectile) followed [113]. At Mach 1.1, the influence of the ground was felt by the projectile at over 5 projectile diameters from the ground (the highest $h / d$ looked at being 8 ), with the normal positive force - caused by the shock reflection from the ground - slightly increasing, before changing sign and becoming increasingly negative with ground clearances less than 1 diameter, broadly in line with the previous findings. This was due to increased high pressure on the forward lower surface, and increased low pressure on the rear lower surface. As a result of this effect the pitching moment also increased rapidly in the nose-up sense. The side force and side moment changes were extremely small. At Mach 1.2, the trends were similar, but all effects are exaggerated, and the projectile felt the influence of the ground at a much lower ground clearance of 3 diameters and below. Results at a clearance of 2 diameters highlighted the potential for sudden spikes in force and moment if shock reflections interact with specific points on the geometry such as where an existing significant flow expansion exists. Because the projectile was spinning, the normal force would manifest itself as a lateral force due to the precession effect, but the actual forces involved are of sufficiently small magnitude to have nowhere near the influence that ground effect was previously observed to have for a projectile travelling at a much higher Mach number [114], and the trajectory would likely remain largely unaltered over any realistic distance.

\section{Supersonic to hypersonic}

\subsection{Sled and launch vehicles}

This paper has already presented findings related to rocket sled testing facilities and geometries which operate in ground effect regardless of the main intention of the tests - in this section some additional studies are described that further add to the body of knowledge. Further wind tunnel tests on the rocket sled configurations real-world tested in the 1960s for the Holloman high speed track were described by Rhudy and Corce [35]. Amongst many parameters tested was the effect of the ride height of the main sled body above the rail (and ground) by adjusting the slipper height, and the influence of the nose shape (i.e. a sharp cone vs. a more blunt tip).

Figs. 25-27 reproduce results for the Mach number range 1.5-3 for normal force (lift) coefficient, pitching moment coefficient, and axial force (drag) coefficient for a narrow range of ground clearances, $h / d=0.5,0.55$ and 0.6 . The change in ground clearance here represents a difference of just $7.6 \mathrm{~mm}$ for the wind tunnel model, yet Fig. 25 indicates up to a 30\% difference between the two extreme ground clearances for lift force at around Mach 2. All three clearances exhibit similar general trends of markedly increased lift between Mach 1.5 and 2, followed by a distinct drop in lift to Mach 2.5. The very high sensitivity to ground clearance and freestream Mach number is evident also in the $h / d=0.55$ case which reverses trends between Mach 1.5 and 2 - indicative, as seen with projectiles and other similar geometries, of shock impingement on a sensitive region, for instance exactly coinciding with an expansion region where the cone meets the main body. As could be expected, the changes become less dramatic the higher the Mach number, as shocks will tend to reflect fewer

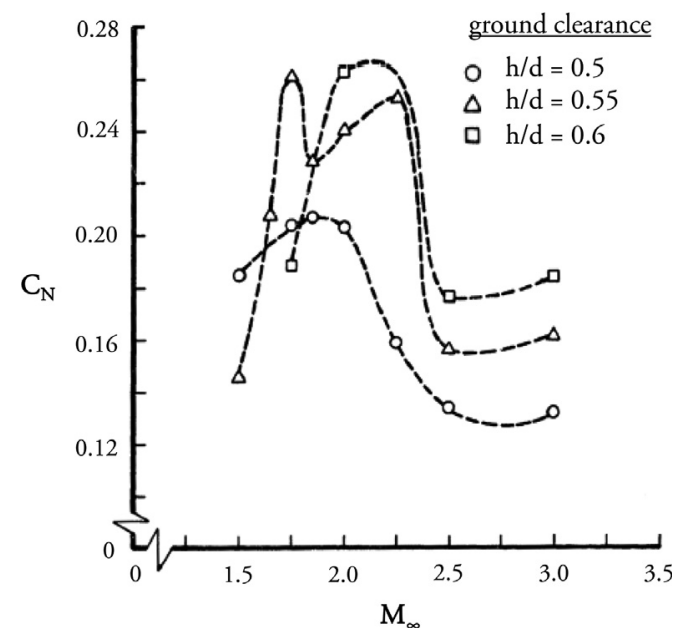

Fig. 25. Normal (lifting) force coefficient on a rocket sled/slipper/rail arrangement from a wind tunnel scale test of varying ground clearances (slipper heights). Adapted from [33]. 


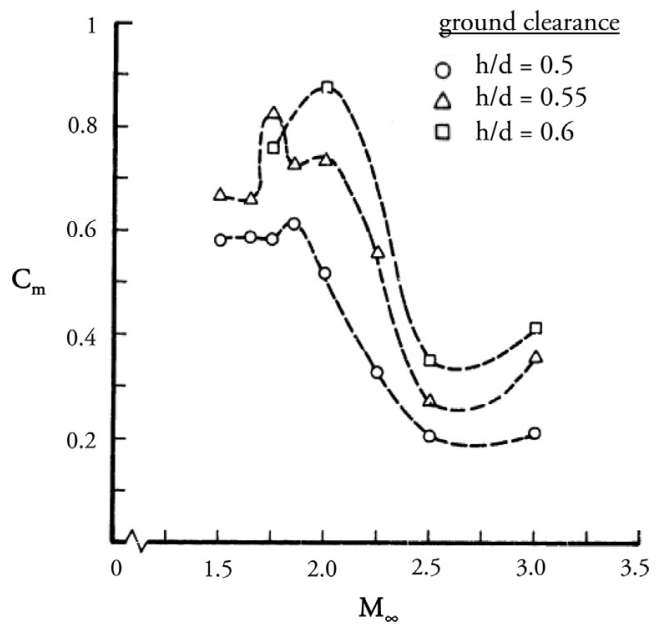

Fig. 26. Pitching moment coefficient on a rocket sled/slipper/rail arrangement from a wind tunnel scale test of varying ground clearances (slipper heights). Adapted from [33].

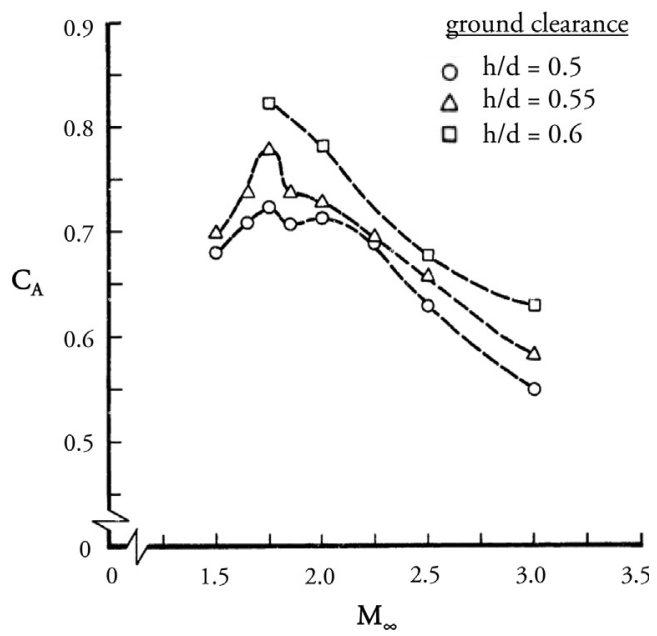

Fig. 27. Axial force coefficient (drag) on a rocket sled/slipper/rail arrangement from a wind tunnel scale test of varying ground clearances (slipper heights). Adapted from [33].

times onto the body, but it can be noted that the difference in lift coefficient between $h / d=0.5$ and 0.6 at Mach 3 is still above $25 \%$. This not only re-iterates how crucial fidelity in the wind tunnel is, but also how significantly the forces (and therefore response) can change for a supersonic object in ground effect, particularly if it were unconstrained.

The corresponding forces and moments from Mach 3.5 to 5.5, obtained in a similar but separate experimental campaign [119], exhibited more gentle, predictable force variations, albeit with some significant changes in that range that can only be attributed to the "switch" of reflected shock interactions on one part of the body to another.

Pitching behaviour, reproduced in Fig. 26, is more predictable in terms of trends across the ground clearances but is greatly affected by Mach number as anticipated from the lift behaviour. The axial (drag) force, from Fig. 27, is the least sensitive to the variables, but as with lift and pitching moment the $h / d=0.55$ case clearly experiences a shock-related anomaly at around Mach 1.75 . Fig. 28 indicates that changing the nose shape will not dramatically change lifting characteristics (the shock angles would not be markedly different for a blunted nose of half the length as they propagate to the far field), but the effect noted at Mach 1.75 is exaggerated, indicating that the shock reflection impingement

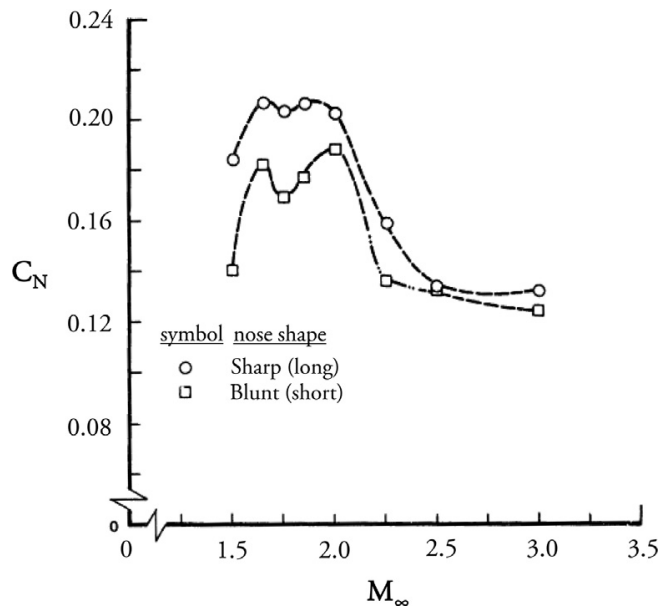

Fig. 28. Normal force coefficient (lift) on a rocket sled/slipper/rail arrangement from a wind tunnel scale test of varying nose shapes for a fixed ground clearance (slipper heights).

Adapted from [33].

location was altered by the changed wave shape formation at the nose.

Given the propensity for excessive lift and the difficulties in balancing this in the face of considerable variation across the Mach range and the proven sensitivity to nearly every variable, the development of an electromagnetically levitated test track at Holloman was instigated to bypass the expensive maintenance and repair costs induced by rail gouging in the supersonic to hypersonic regime. The sensitivity of the levitation behaviour due to changing and potentially sensitive aerodynamic forces due to shock-ground reflections, particularly in the transonic-to-lowsupersonic range, was of concern for long-term fatigue and repeatability of tests. Schlieren photography was used to visualise these shock reflections [115] and help guide the development of a preferred guideway within which the coils are embedded to produce the magnetic field. The concern was that excessive aerodynamic forces caused by shock reflections would cause the sled to depart from the guideway (i.e. lift out of the controlled field), and extensive experimentation was pursued in concert with CFD which was substantially validated as a result. Using focusing schlieren to illustrate the way in which the bow shock from the nosecone reflected into the body downstream from the front slipper of the sled [115], the investigators noted that this interaction affects the pitch rate of the test payload as it impacts to the target zone shortly after separating from the sled which continues under the impact zone and is brought to rest. This therefore can have an influence on the test results obtained. The schlieren also highlights shock-shock interactions where the bow wave intersects the normal shock infront of the slipper front wedge, which leads to the convoluted shock front observed more keenly in 3-dimensions in numerical studies [116].

The complexity of the payload/sled/slipper/rail arrangement was highlighted by Lofthouse et al. [116]. RANS CFD using Fluent was described at considerable length. With mesh cell counts in the hundreds of thousands to low millions - despite local shockcapturing refinement - and some one-cell gaps between the slipper and the rail, the results may have been significantly out of concert with the physical realities due to excessive dissipation of waves on reflection and the negation of the boundary layer. Nevertheless, useful visualisation and pressure correlation of the shock/sled/slipper interactions was shown, indicating the complexity of the shock front which forms at Machs 2 and 3 due to the ground reflection and numerous interactions with the sled 
arrangement, and both air and helium were investigated as test gases. The need for proper transient numerical investigation of shock interaction phenomena is pressed by the authors, and a high-resolution, unsteady simulation would be readily possible with today's computing power, extending to realistic thermal modelling in addition.

Praharaj [117] outline the clear advantages of simulation: relatively inexpensive testing of different gas compositions (in this instance helium and air), multiple potential design configurations, and aerodynamic effects likely to be encountered in subsequent testing. The ability to understand the underlying causes of force and moment variations is a key capability of any CFD code and in ground effect may be particularly useful due to concerns over the validity of experimental testing using anything other than a sled/rail arrangement.

A proprietary viscous RANS code was used with algebraic and $k-\varepsilon$ turbulence models, and the highly 3-dimensionality of the flowfield was discussed with reference in particular to canard interactions at mid-supersonic Mach numbers. The fully structured meshes proved highly capable at reproducing real-world pressure data from the surface of the sled/slipper/rail/canard arrangement, and contour plots indicated reasonable resolution of the shock reflections under the sled body. Six specific shock reflection/interactions were noted, and a significant side force acting on each side of the sled (i.e. on each rail), and it was proposed that CFD is an effective design tool for strakes and canards to provide corrective and restoring moments to avoid destabilisation. Moreover, the ability to predict precise loads on the structure has far-reaching implications for this particular application, and the likelihood of greatly reduced material failure in actual tests as a result.

In discussing canard design for simple sled arrangements such as those originally used at Holloman in the 1960s, Rigali and Feltz [118] describe the small but effective nose-cone canard reduces the normal force, across Mach numbers from 2 to 5 , to within \pm 0.1 of zero, as opposed to the original design producing a $C_{L}$ in excess of 0.2 . However, the canard version does promote a greater change in load when traversing the Mach number regime, including force reversal from Mach 2 to Mach 3 (and another reversal between Mach 6 and 10).

Meinke et al. [120] mention the shock-reflection issue for a proposed electromagnetic launch system for a fully enclosed tube scenario, stating that preliminary inviscid and viscous CFD indicated that the shock reflections from the wall had important implications for the vehicle stability. Given that the simulations were for a 1/100th of an atmosphere, it is doubtful that their choice of Fluent as a CFD code would have been suitable for such conditions, and as a result they used air as a medium for Mach 2 tests. Compromises in ground clearance (i.e. an increase) had to be made to avoid divergence due to the complexities of the multiple shock/body interactions from wall reflections - there were 4 or more interactions at Mach 2, and not until Mach 7 did the wall-reflected shock clear the tail of the body.

\subsection{Projectiles}

A study to examine the propagation or otherwise of sonic booms in water from the close overflight of a projectile at Mach 5.7 and 2.7 (the former being fast enough to produce a corresponding shock in the water) was conducted by Intrieri and Malcolm [121]. Shadowgraph pictures revealed the characteristic regular reflection of the bow and recompression shocks from the water surface, but the ground clearance was approximately 5 diameters and therefore the reflections passed into the far wake and would have had no influence on the projectile trajectory as the surface pressure distribution would be unaltered. The peak pressure at the water surface due to overflight exhibited a log-linear increase over the range of clearances tested, none of which were low enough to produce a reflection into the near-wake.

In examining lower ground clearances for a military projectile, a series of investigations by Doig et al. provided extensive data on flow around a Mach 2.4 NATO $5.56 \mathrm{~mm}$ projectile in close proximity to a ground plane [122]. The investigation was conducted along similar lines as that described in Section 3 for a Mach 1.1 and 1.2 projectile of similar shape - live range experiments coupled with scaled, nonspinning wind tunnel models and steady-state CFD. An example live-range schlieren image was shown in Fig. 23(b).

Multiple ground clearances from $h / d=2$ to 0.2 were tested, and the three-dimensional propagation and reflection of the shock waves were considered in detail. The behaviour of the flow in the near wake was also studied as ground clearance was reduced, making it the only notable study that has explicitly examined wake behaviour in supersonic ground effect in detail. It was found that RANS modelling was capable of closely reproducing the shock reflection patterns of the quasi-steady projectile flow state, but that extensive local refinement of the mesh for shock-capturing was required to sustain more than one reflection between the projectile and the ground.

The authors identified three types of shock reflection/interactions; a type A interaction (in this instance for $h / d>1.3$ ), whereby the reflection of the projectile bow shock from the ground plane met only the far wake, and was found to exert little influence on the aerodynamic forces and moments of the body. A type B case $(1<h / d<1.3)$ was defined as featuring a strong interaction between the reflected bow wave and the near wake of the projectile, at which point the aerodynamic forces and moments were found to experience a change from freestream conditions, with drag decreasing due to increased pressure in the base region. A type $C$ case $(h / d<0.75)$ produced a strong normal force acting on the projectile, which, due to the body's rate of spin, would produce a lateral precession. Drag increased significantly as the wake was thickened and drawn toward the ground, and a weak lateral force was also discovered as ground clearance was reduced, which, again as the projectile is spinning, would produce a small influence on the vertical movement of the projectile relative to the ground plane. Fig. 29 shows comparisons of numerical and experimental schlieren for a Type A case $(h / d=1.77)$ and a Type $C$ case $(h / d=0.75)$. The forces and moments are shown in Figs. 32 and 33 , indicating the magnitude of the normal force is extensive at low ground clearances as experienced by the similar rocket sled geometries, and the pitching tendency of the projectile changes sign in the region $h / c<1$.

The projectile was discovered to experience a marked pitching moment, initially nose-downwards at the high end of type $C$ reflection interactions, then more strongly nose-up with continued decreasing ground clearance, as the pressure distribution over the rear of the projectile was increasingly distorted by the build up of high pressure behind the series of shock/ground interactions. Reflections downstream of the projectile interacted heavily on the wake, distorting its structure and drawing it initially up (type A and B cases), then strongly downwards to interact with the ground in the far field (type C cases). These interactions were shown to be complex and highly three-dimensional, and CFD provided a wealth of information that was not gleaned from the original live-range or wind-tunnel experiments: the detailed wake profiles, wake flow structures and the reason for their deflections, the ground pressure distributions describing the "footprint" of the shocks there, visualisation of the propagation of the shocks and their reflections in three dimensions. For example, Fig. 30 shows patterns of shock reflections in planes normal to the projectile, indicating the reflecting wave behaviour and the influence on the wake for a Type $C$ interaction. The flowfields are reminiscent of the inviscid store release work of Marconi [14], previously mentioned in the 

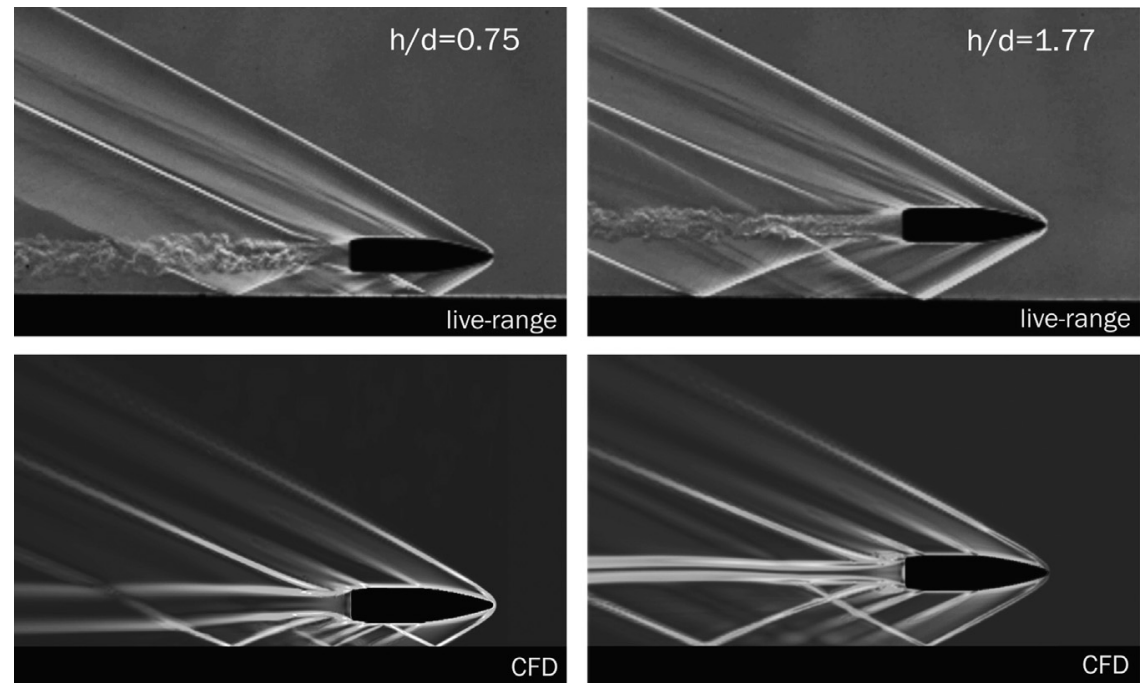

Fig. 29. Comparison of numerical schlieren and live-range experiments for a projectile at Mach 2.4 and $h / d=0.75$ and 1.77 [122].

1

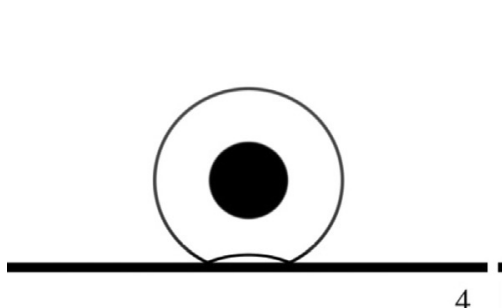

4

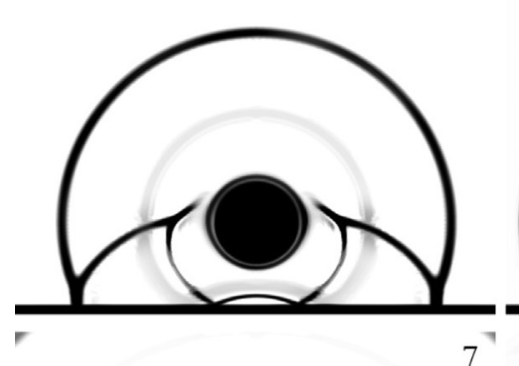

$7^{7}$
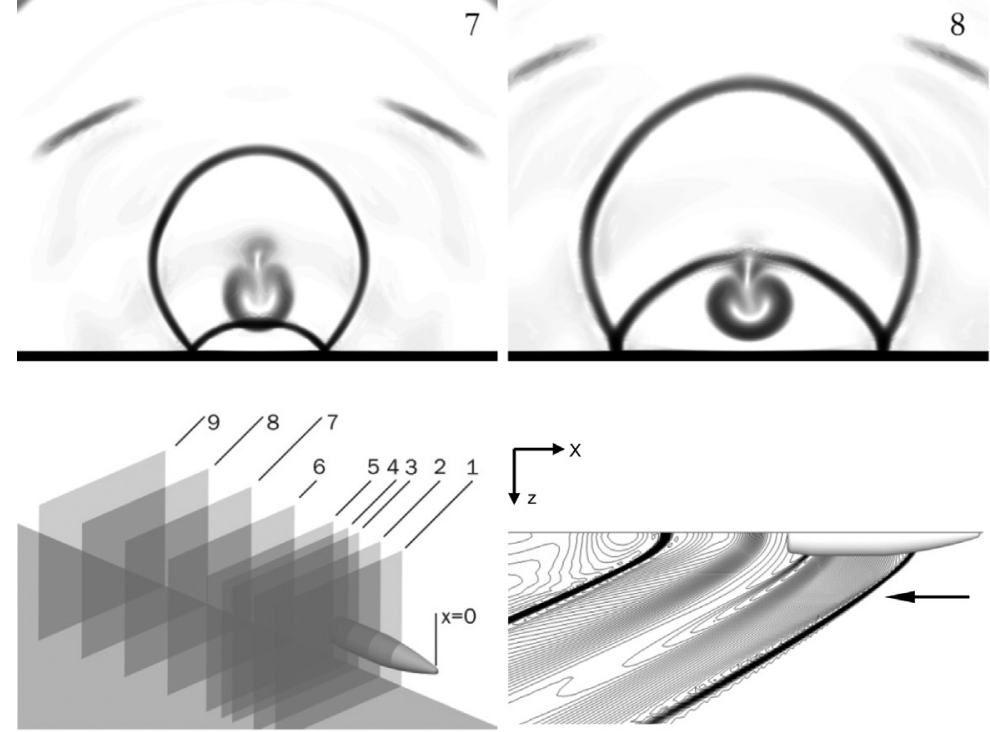

2

3
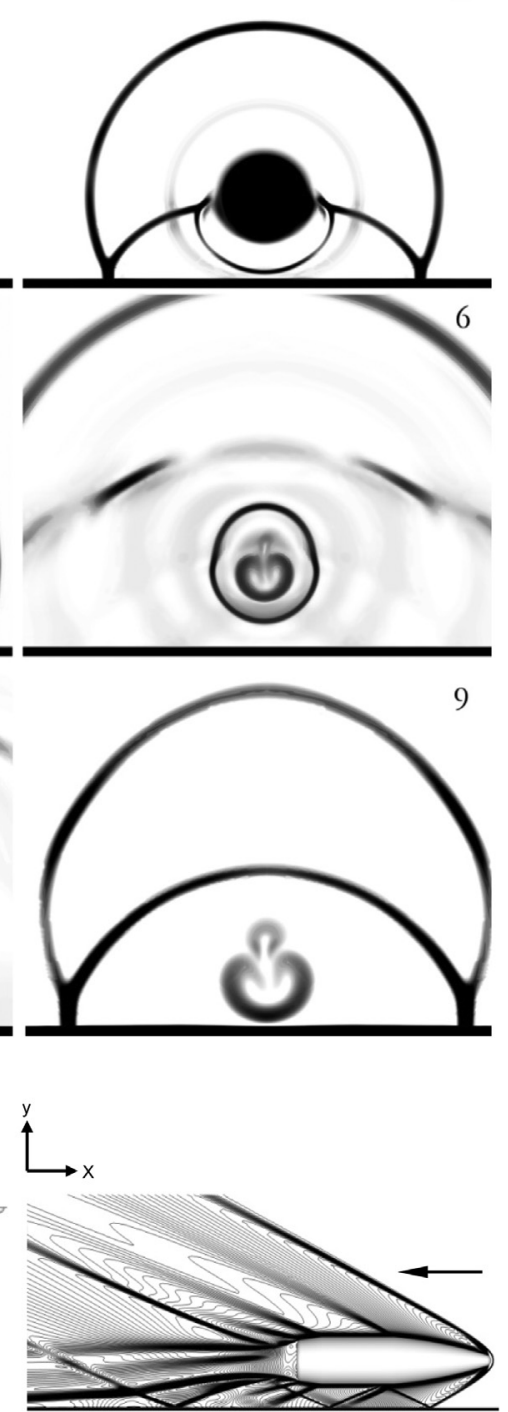

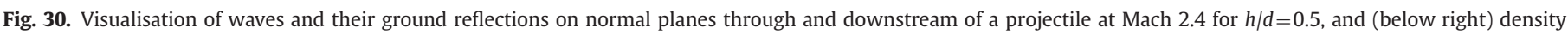
contours on the ground plane and projectile mid-plane. 

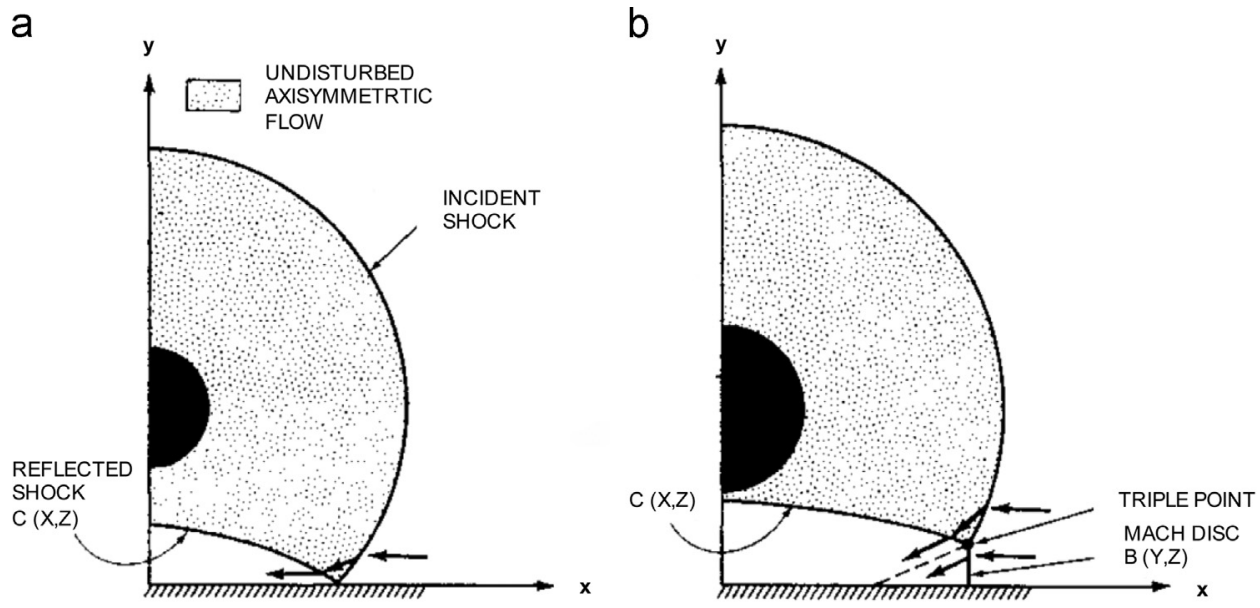

Cross-sectional regular and Mach reflection

Fig. 31. Sketch of waves and reflection produced by an inviscid simulation of a weapon store in surface proximity, (a) Regular reflection and (b) mach reflection. Source: [14].

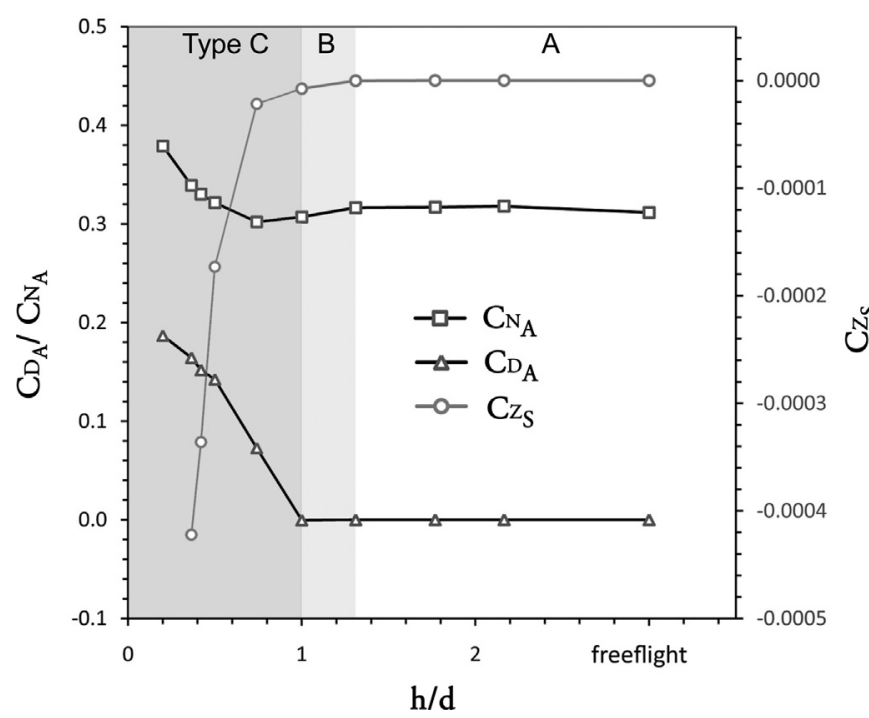

Fig. 32. Normal, drag and side force acting on a projectile at Mach 2.4 for varying ground clearances.

Data: [122].

opening section and reproduced in Fig. 31. Pathlines indicating wake behaviour on the vertical mid-plane of the projectile are shown in Fig. 34, indicating the distortion of the vortex loop with reducing clearance as the low pressure of the "boat-tail" expansion/reflection region draws the wake sharply towards the ground plane, simultaneously entraining greater flow from the upper portion of the wake.

\section{Conclusions}

The study of ground effect aerodynamics in the transonic and supersonic regime is an active area that shares flow similarities with several other fundamental and applied research areas in fluid mechanics. Considerable advances have been made in understanding flowfields featuring shock interactions with a ground plane and their reflection back onto the body which generated them one or more times. However, a feature of the majority of

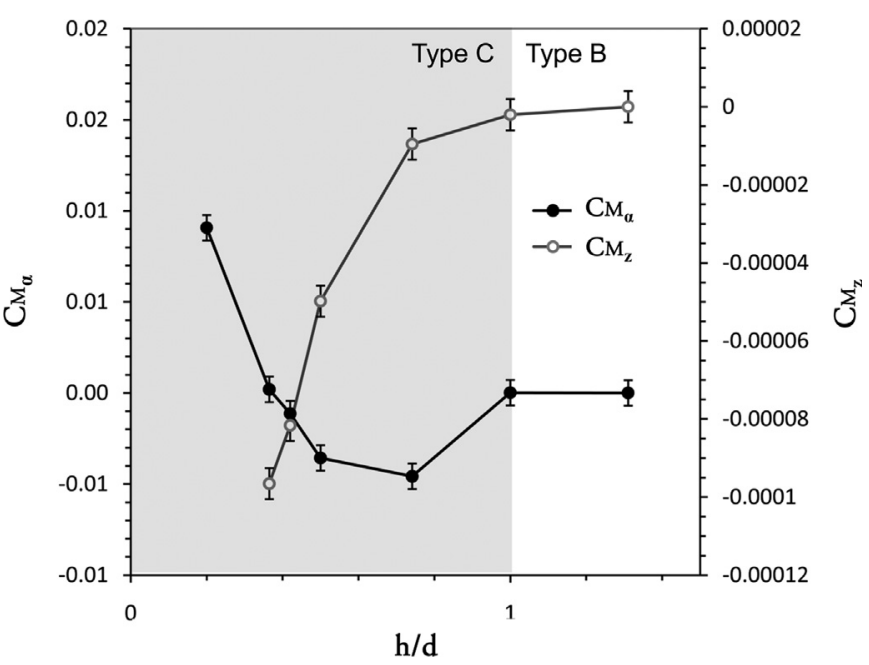

Fig. 33. Pitching and yaw moments acting on a projectile at Mach 2.4 for varying ground clearances. Data: [122].

studies described here is that they have been conducted at different Reynolds numbers, in different flow conditions, and for many different bodies. Therefore, there is yet to emerge a cohesive field that can advance on specific non-design goals for a more scientific understanding of some of the pertinent phenomena such as shock waves reflecting from moving ground planes with different boundary layer conditions.

It has been established that there are several ways to study high speed ground effect problems with controlled, repeatable experiments, but only a sled arrangement or close equivalent whereby the object is able to move over a stationary ground plane (with a sufficiently long ground run for the flowfield to adjust) - is able to produce data which could be considered accurate in all conditions, discounting Reynolds-scaling effects which are a problem for any small-scale aerodynamic testing. Such apparatus and testing is extremely expensive, prohibitively so for most studies, and therefore use of an elevated ground plane or a mirror image symmetry model in a wind tunnel is common. The latter method has been shown to provide results with very good equivalency to those which could theoretically be obtained using a moving 

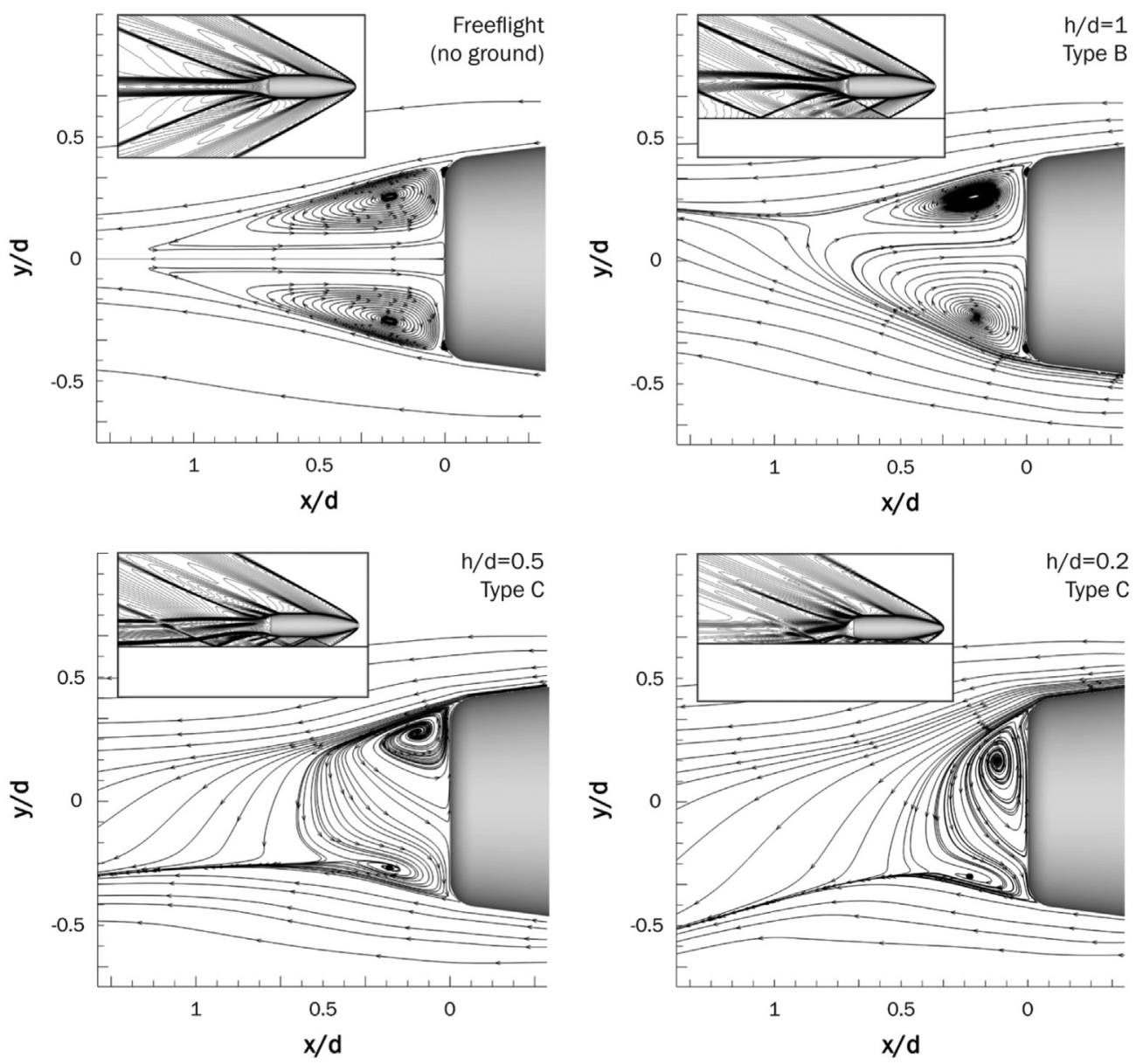

Fig. 34. Wake and vortex ring structure behind a Mach 2.4 projectile for various ground clearances.

Source: [122].

ground at transonic and supersonic conditions, while an elevated ground has a more limited range of effectiveness. Ground effectspecific drawbacks of any tunnel-based method include extra blockage from the additional geometry.

As a result of the difficulties in accurately producing the ground boundary accurately, one obvious lack in the field is comprehensive, high-fidelity experimental data. In many aerodynamic applications, CFD is now generally accepted as the primary design tool supported by experiments rather than the traditional vice-versa, yet it remains essential that simulations be effectively validated. At the moment the ground effect researcher has very little to choose from above Mach 0.5, and the available tests are not always well documented. There is virtually no quantitative data regarding wakes for such flows. For numerical work involving complex multiple flow shock/BL interactions for moving surfaces, and for discerning the appropriate turbulence modelling approach or off-surface mesh resolution, etc., the pressing need for reliable, multi-technique reference data is clear. It is therefore proposed that thorough, highquality wind tunnel testing - most likely using the symmetry method as the best-available low-cost proxy - of appropriate wing sections and applicable, relevant geometry such as ogives, conecylinder arrangements, and simple wing-body arrangements suitable for generic re-usable spacecraft be conducted as a resource for the ground effect aerodynamics community.

Across rail-based vehicles, rail sleds themselves, projectiles, rocket/ jet cars and wings and aircraft in ground effect at transonic speeds, the common findings were that the formation of shock waves between the object and ground had a marked effect on the aerodynamic forces and moments. In many cases, the more complex the geometry was, the more complex the effects observed. In the case of long slender bodies, an initial neutral or negative normal force configuration is likely to become a strong positive normal (usually lifting) force as shocks produce a build-up of high pressure under the body, and effects on wings are highly dependent on the camber and incidence. Pitching characteristics can also change sign and be affected considerably, depending on the location of the centre of gravity relative to shock formation. All bodies reported in studies seemed to be relatively sensitive to ground clearance (and the height-to-length/chord ratio), incidence, and Mach number. An earlier transonic drag rise due to a lower supercritical Mach number is a common feature.

At fully supersonic to hypersonic Mach numbers, the effects are more predictable as oblique shock reflection from the ground interacts once or more with the body in question. Here, the ground clearance for a given body with a particular shock angle is the primary variable as the location of shock impingement determines the pitching behaviour, and the number of interactions determines how much additional normal force can be produced by ground proximity. The normal force for a spinning projectile would induce a lateral movement, unlike the lift it would impart on a conventional body.

Regardless of Mach number involved, the unique nature of shock/moving ground interactions is not well described in any literature, though some analogous flowfields may be found in other areas of research.

The above points also bolster evidence presented elsewhere in this paper indicating the need for more comprehensive numerical studies in the field, exploring the nature of shock/ground interactions more thoroughly and pursuing transient and LES-based approaches for increased physical accuracy, particularly relating to 
wake behaviour and shock/BL interaction. The work described in this review describes applied studies with the implication that there is a lack of fundamental work into the nature of shock/surface interactions in a moving reference frame appropriate to the field; there are undoubtedly many interesting wave reflection phenomena which will complement the findings of the multitude of existing studies into shock/shock and shock/BL interactions. At the mid-supersonic to hypersonic range, heat transfer is evidently a major concern in the presence of shock/body interactions, and RANS has traditionally been very weak in this area [123], further pressing the case for more advanced techniques to be applied.

The majority of studies conducted to date have been for bodies which are aligned with the flow and in effectively steady-state conditions. The effects of acceleration and deceleration should also become a focus of investigation as an inherent feature of many applications. The precise characteristics of flow at the onset of shock formation and reflection from the ground plane should be explored further, as for any body this appears to be the most destabilising moment.

It is inevitable that considerable further work in transonic and supersonic ground effect - particularly in the fields of high-speed Maglev transportation and rail or tube launch vehicles - will be conducted in the near future. Therefore it is proposed that authors of technical works on the subject specifically include "ground effect" in their keywords and, where appropriate, adopt a standard means of reporting the ground clearances in non-dimensional terms ( $h / d, h / l, h / c$, etc.). In this way, a more consistent body of work can be effectively shared amongst researchers as the body of work in high speed ground effect aerodynamics continues to grow.

\section{Acknowledgements}

The input of Dr. Tracie Barber, Dr. Andrew Neely, and Prof. Eddie Leonardi into much of the author's own technical work described here is gratefully acknowledged, as is the support of Dr. Harald Kleine and Dr. David Myre. Some original results not previously reported stem from work part-funded by the American Australian Association, the Royal Aeronautical Society, and the UNSW Faculty of Engineering.

\section{References}

[1] Rozhdestvensky KV. Wing-in-ground effect vehicles. Prog Aerosp Sci 2006;42(3):211-83.

[2] Powell J, Maise G, Paniagua J, Rather J. Maglev launch and the next race to space. In: IEEE aerospace conference proceedings. Big Sky, USA, 2008. p. 1-20.

[3] Schetz JA. Aerodynamics of high speed trains. Annu Rev Fluid Mech 2001;33:371-414.

[4] Kleine H, Oakes B, Pratt H, Neely AJ, Barber TJ. Shock wave interaction with gaseous plumes and flames. In: 29th International congress on high-speed imaging and photonics proceedings, ICHSIP-29; 2010. p. 1-6.

[5] Doig G, Johnson Z, Mann R. Interaction of shock tube exhaust flow with a non-pre-mixed flame. J Visual 2013;16(3):173-6.

[6] Altmann J. Acoustic weapons-a prospective assessment. Sci Glob Secur 2001;9(3):165-234.

[7] Maskalik AI, Rozhdestvensky KVA. View of the present state of research in aero- and hydrodynamics of ekranoplans. In: RTO AVT symposium on fluid dynamics problems of vehicles operating near or in the air-sea interface, Amsterdam; 5-8 October 1998.

[8] Krothapalli A, Rajkuperan E, Alvi F, Lourenco L. Flow field and noise characteristics of a supersonic impinging jet. J Fluid Mech 1999;392 (1):155-81.

[9] Doig G. Compressible ground effect aerodynamics (Doctoral dissertation), University of New South Wales; 2009.

[10] Mach E, Wosyka J. Über einige mechanische Wirkungen des elektrischen Funkens. Sitz. Akad. Wiss Wien II. Abth. 1875;72:44-52.

[11] Szwaba R, Doerffer P, Namieśnik K, Szulc O. Flow structure in the region of three shock wave interaction. Aerosp Sci Technol 2004;8(6):499-508.

[12] Ben-Dor GA. State-of-the-knowledge review on pseudo-steady shock-wave reflections and their transition criteria. Shock Waves 2006;15(3-4):277-94.

[13] Ewald BF. Wind tunnel wall corrections (la Correction des effets de paroi en soufflerie); 1998. AGARD-AG-336.
[14] Marconi F. Shock reflection transition in 3-dimensional steady flow about interfering bodies. AIAA J 1983;21(5):707-13.

[15] Hornung H. Regular and Mach reflection of shock waves. Annu Rev Fluid Mech 1986; 18:33-58.

[16] Delery JM. Shock wave/turbulent boundary layer interaction and its control. Prog Aerosp Sci 1985;22:209-80.

[17] Henderson LF. The reflexion of a shock wave at a rigid wall in the presence of a boundary layer. J Fluid Mech 1967;30(4):699-722.

[18] Ben-Dor G, Takayama K. The phenomena of shock wave reflection-a review of unsolved problems and future research needs. Shock Waves 1992;2(4): $211-23$.

[19] Fago B, Lindner H, Mahrenholtz O. The effect of ground simulation on the flow around vehicles in wind tunnel testing. J Wind Eng Ind Aerodyn 1991;38:47-57.

[20] Weiselsberger C. Wing resistance near the ground; 1922. NACA TM-77.

[21] Barber TJ, Leonardi E, Archer RD. A technical note on the appropriate CFD boundary conditions for the prediction of ground effect aerodynamics. Aeronaut J 1999;103(1029):545-7.

[22] Barber TJ, Leonardi E, Archer RD. Causes for discrepancies in ground effect analyses. Aeronaut J 2002;106(1066):653-7.

[23] Sowdon A, Hori T. An experimental technique for accurate simulation of the flowfield for wing in surface effect craft. Aeronaut J 1996:215-22 (June/July).

[24] Willemsen E. High Reynolds number wind tunnel experiments on trains. J Wind Eng Ind Aerodyn 1997;69:437-47.

[25] Baker CJ, Brockie NJ. Wind tunnel tests to obtain train aerodynamic drag coefficients: Reynolds number and ground simulation effects. J Wind Eng Ind Aerodyn 1991;38(1):23-8.

[26] Baker CJ, Humphreys ND. Assessment of the adequacy of various wind tunnel technique to obtain aerodynamic data for ground vehicles in cross winds. J Wind Eng Ind Aerodyn 1996;60:49-68.

[27] Fink MP, Lastinger JL. Aerodynamic characteristics of low-aspect-ratio wings in close proximity to the ground, 1961 NASA Technical Note D-926.

[28] Doig G, Barber TJ, Neely AJ, Myre DD. Experimental validation as an integral component of computational fluid dynamic research. ANZIAM J 2010 265-79.

[29] Barber T], Doig G, Beves C, Watson I, Diasinos S. The synergistic integration of CFD and EFD for ground effect aerodynamics studies. Proc IMechE Part G: J Aerosp Eng 2012;226(6):602-19.

[30] Selescu R. Adapting a blowdown type wind tunnel for ground effect simulation tests. In: Proceeding of the 9th WSEAS international conference on automation and information (ICAI 2008). p. 24-6.

[31] Nakata D, Yajima J, Nishine K, Higashino K, Tanatsugu N, Kozu A. Research and development of high speed test track facility in Japan. In: Proceedings of the 50th AIAA aerospace sciences meeting 2012, Nashville, Tennessee. p. 1-11.

[32] Nakata D, Kozu A, Yajima J, Nishine K, Higashino K, Tanatsugu N. Predicted and experimented acceleration profile of the rocket sled. Trans Jpn Soc Artif Intell Aerosp Technol Jpn 2012:10.

[33] Strike Jr. WT, Lucas EJ. Evaluation of wind tunnel tests on Afmdc monorail cone-and spike-nose sled configurations at Mach numbers from 2.0 to 5.0; 1968.

[34] Minto DW. CTEIP funded advances in hypersonic testing at the Holloman High Speed Test Track. In: Proceedings of the 24th AIAA aerodynamic measurement technology and ground testing conference; 2012.

[35] Rhudy RH, Corce JD. Static force and moment tests of the holloman narrowgage rocket sled at Mach numbers from 1.5 to $4.0 ; 1975$.

[36] Lamb JL. Critical velocities for rocket sled excitation for rail resonance. Johns Hopkins APL Tech Dig 2001;21(3):448-58.

[37] Knox EC, Myers JE, Cummings J. Development of ambient hypersonic ground testing. In: Proceedings 20th AIAA advanced measurement and ground testing technology conference; 1998.

[38] Turnbull D, Hooser C, Hooser M, Myers J. Soft sled test capability at the holloman high speed test track; 2010.

[39] Hegedus MC, Mendenhall MR, Perkins Jr SC, Stremel PM, Faltz, JA. Engineering analysis for rocket sled aerodynamics. AIAA; 2006. p. 664.

[40] Doig G, Barber TJ, Leonardi E, Neely AJ, Kleine H. Methods for investigating supersonic ground effect in a blowdown wind tunnel. Shock Waves 2008;18 (2):155-9.

[41] Krupovage DJ, Mixon LC, Pokorny OT. Wind-tunnel and full-scale forces on rocket sleds. J Spacecr Rocket 1967;4(10):1346-51.

[42] Krupovage DJ. Two and three dimensional transonic model test results obtained at the Holloman test track facility; 1978.

[43] Carriage K, Young J, Kleine H, Hiraki K. Reynolds-averaged Navier-Stokes computation of transonic projectiles in ground effect. In: Australasian fluid mechanics conference, Launceston, Australia; 2012. p. 1-4.

[44] Briggs RA, Korkegi RH. The hypersonic slipper bearing - a test track problem. J Spacecr Rocket 1969;6(2):210-2.

[45] Korff $\mathrm{WH}$. The aerodynamic design of the goldenrod-to increase stability, traction, and speed. Training 1966:01-21.

[46] Diasinos S, Doig G, Barber TJ. The effects of simplifications on isolated whee aerodynamics, Int J Wind Eng Ind Aerodyn June 2013 (in press, revision submitted December 2013).

[47] Bork CE. Aerodynamic development of the Buckeye Bullet 3 electric landspeed vehicle (Doctoral dissertation). Ohio State University; 2012.

[48] Torda TP, Uzgiris SC. Blue flame - a land speed record vehicle. Mech Eng 1970;92(7):9-18. 
[49] Doig G, Neely AJ, Barber TJ, Leonardi E. The use of thermochromic liquid crystals to investigate flow field interactions with the ground around a supersonic land speed record vehicle. In: Proceedings of the 13 international heat transfer conference, Sydney; 2006.

[50] Torda TP, Morel TA. Aerodynamic design of a land speed record car. J Aircr 1971:8(12):1029-33.

[51] Noble R, Tremayne D. Thrust (text). Partridge Press; 1-8522-5268-5.

[52] Major I. R \& D ranges and test facilities: summary of capabilities. United States Army Test and Evaluation Command AD-A265 938; 1990.

[53] Ayres R, Thrust SSC. Environmental testing. Environ Eng 1996;9(2):28-30.

[54] Haddleton GP. Use of high-speed photography and associated techniques as a measurement tool in the research, development, test, and evaluation of weapons systems and armaments. In: High-speed photography and photonics: 21st international congress. International Society for Optics and Photonics; 1995. p. 782-91.

[55] Kleine $H$, Hiraki K, Oakes B, Young J, Kusano $H$, Iritani Y, Projectiles in transonic ground effect. In: Proceedings of the 29th international congress on high-speed imaging \& photonics; 2011.

[56] Doig G, Barber TJ, Neely AJ, Myre DD. Aerodynamics of an aerofoil in transonic ground effect: methods for blowdown wind tunnel scale testing. Aeronaut J 2012;116(1180):599-620.

[57] Bushnell DM. Scaling: wind tunnel to flight. Annu Rev Fluid Mech 2006:38:111-28.

[58] Pettersson K, Rizzi A. Aerodynamic scaling to free flight conditions: past and present. Prog Aerosp Sci 2008;44(4):295-313.

[59] Schuster DM. Aerodynamic measurements on a large splitter plate for the NASA Langley Transonic Dynamics Tunnel. National Aeronautics and Space Administration, Langley Research Center; 2001.

[60] Dudley JG, Ukeiley L. Numerical investigation of a cylinder immersed in a supersonic boundary layer. AIAA J 2012;50(2):257-70.

[61] Holloran M, O’Meara S. Wing in ground effect craft review (No. DSTO-GD-0201) Canberra (Australia): Defence Science and Technology Organisation; 1999.

[62] Zhang X, Toet W, Zerihan J. Ground effect aerodynamics of race cars. Appl Mech Rev 2006:59:33-49.

[63] Katz J. Aerodynamics of race cars. Annu Rev Fluid Mech 2006;38:27-63.

[64] Schetz JA. Aerodynamics of high speed trains. Annu Rev Fluid Mech 2001:33:371-414

[65] Ahmed MR, Sharma SD. An investigation on the aerodynamics of a symmetrical airfoil in ground effect. Exp Therm Fluid Sci 2005;29:633-47.

[66] Dominy RG. Aerodynamics of Grand Prix car. Proc Inst Mech Eng 1992;206:267-74.

[67] Keogh J, Doig G, Diasinos S. Flow compressibility effects around an openwheel racing car. Aeronaut J 2013 (in press).

[68] Keogh J, Doig G, Diasinos S. The influence of compressibility effects in correlation issues for aerodynamic development of racing cars. In: 18th Australiasian fluid mechanics conference, Launceston; 3-7 December 2012.

[69] Doig G, Barber TJ, Leonardi E, Neely AJ. The onset of compressibility effects for aerofoils in ground effect. Aeronaut J 2007:111(1126):797-806.

[70] Doig G, Barber TJ, Diasinos S. Implications of compressibility effects for Reynolds-scaled testing of an inverted wing in ground effect. Int J Aerodyn September 2013 (in press).

[71] Doig G, Barber TJ, Neely AJ. The influence of compressibility on the aerodynamics of an inverted wing in ground effect. ASME J Fluids Eng $2011 ; 133(6): 1-12$.

[72] Glauert H. The effect of compressibility on the lift of an aerofoil. Proc R Soc London 1928;CXVIII:113-9.

[73] Göthert BH. Plane and three-dimensional flow at high subsonic speeds (extension of the Prandtl rule). NACA TM 1946:1105.

[74] Lock A. Computational fluid dynamics development of the JCB DIESELMAX land speed record vehicle. Training 2009:01-26.

[75] Shih TH, Liou WW, Shabir A, Yang Z, Zhu J. A new k-e Eddy Viscocity model for high reynolds number turbulent flows. Comput Fluids 1995;24 (3):227-38.

[76] Oberoi R, Chakravarthy S, Fredrick W, Glessner PT. Using CFD to design the American challenger rocket car. SAE 2006:206-013660.

[77] Goldberg U, Akdag V, Palaniswamy S, Oberoi R, Bachchan N, Glessner P, et al. CFD analysis of the American challenger rocket car. In: SAE 2006 world congress \& exhibition, vehicle aerodynamics. p. 251-8.

[78] Wang S. Aerodynamic aspects of land speed record cars (Honours Thesis) University of New South Wales; 2012.

[79] Spalart P, Allmaras S. A one-equation turbulence model for aerodynamic flows. AIAA Paper; 1992; 92-0439.

[80] Ackroyd J. Thrust 2 - design of the world land speed record car. Proc Inst Mech Eng 1985;199:239-64.

[81] Morgan K, Hassan O, Weatherill N. MATHEMATICS - why didn't the supersonic car fly? Math Today - Bull Inst Math Appl 1999;35(4):110-4.

[82] Hwang JP, Doig G, Barber TJ, Neely AJ, Leonardi E. Numerical investigation of the aerodynamics of land speed record vehicles. In: Proceedings of the 17th international shock interaction symposium, Rome; 3-7 September 2006.

[83] Barber JP, Bauer DP. Contact phenomena at hypervelocities. Wear 1982;78 (1):163-9.

[84] Remaki L, Hassan O, Evans BJ, Morgan K. Fluidized sand effect on drag forces for a supersonic vehicle. In: ICCFD 2012, Paris, France; August 22-23, 2012.

[85] Evans BJ, Hassan O, Jones JW, Morgan K, Remaki L. Computational fluid dynamics applied to the aerodynamic design of a land-based supersonic vehicle. Numer Methods Partial Differential Equations 2011;27(1):141-59.
[86] Evans B, Morton T, Sheridan L, Hassan O, Morgan K, Jones JW, et al. Design optimisation using computational fluid dynamics applied to a land-based supersonic vehicle, the BLOODHOUND SSC. Struct Multidiscipl Optim 2013;47(2):301-16.

[87] Raghunathan RS, Kimb HD, Setoguchi T. Aerodynamics of high-speed railway trains. Prog Aerosp Sci 2002:38:469-514.

[88] Bellenoue M, Moriniere V, Kageyama T. Experimental 3d simulation of the compression wave due to train tunnel entry. J Fluids Struct 2002;16 (5):581-95.

[89] Zonglin J, Matsuoka K, Sasoh A, Takayama K. Numerical and experimental investigation of wave dynamic processes in high-speed train/tunnels. Acta Mech Sin 2002;18(3):209-26.

[90] Baron A. Mossi M, Sibilla S. The alleviation of the aerodynamic drag and wave effects of high-speed trains in very long tunnels. J Wind Eng Ind Aerodyn 2001;89(5):365-401.

[91] Kim TK, Kim KH, Kwon HB. Aerodynamic characteristics of a tube train. J Wind Eng Ind Aerodyn 2011;99(12):1187-96.

[92] Hammitt AG. Aerodynamic analysis of tube vehicle systems. AIAA J 1972;10 (3):282-90

[93] Rote DM, Cai Y. Review of dynamic stability of repulsive-force maglev suspension systems. IEEE Trans Magn 2002;38(2):1383-90.

[94] Doig G, Barber TJ, Neely AJ. Progress in understanding transonic and supersonic ground effect. In: 28th international congress of the aeronautical sciences, Brisbane; 23-28 September 2012.

[95] Doig G, Barber TJ, Leonardi E Neely AJ. The aerodynamics of aerofoils and wings in transonic ground effect. In: Proceedings of the 2008 international congress on theoretical and applied mechanics, Adelaide, 1-2.

[96] Dragos L. Numerical solutions of the equation for a thin airfoil in ground effect. AIAA J 1990;28(12):2132-4.

[97] Dragos L, Dinu A. A direct boundary integral equations method to subsonic flow with circulation past thin airfoils in ground effect. Comput Methods Appl Mech Eng 1995;121:163-76.

[98] Maglieri DJ, Huckel V, Parrott TL. Ground measurements of shock-wave pressure for fighter airplanes flying at very low altitudes and comments on associated response phenomena. (NASA-TM-X-611). Hampton VA: NASA Langley; 1961.

[99] Nixon CW, Hille HK, Sommer HC, Guild E. Sonic booms resulting from extremely low-altitude supersonic flight: measurements and observations on houses, livestock and people (No. AMRL-TR-68-52). Wright-Patterson AFB, OH: Air Force Aerospace and Medical Research Laboratory; 1968.

[100] Manci KM, Gladwin DN, Villella R, Cavendish MG. Effects of aircraft noise and sonic booms on domestic animals and wildlife: a literature synthesis (No. NERC-88/29). Fort Collins, CO: National Ecology Research Center; 1988.

[101] Doig G, Barber T, Neely A, Myre DD. Aerodynamics of an aerofoil in transonic ground effect: numerical study at full-scale Reynolds numbers. Aeronaut J 2012;116(1178):407-30.

[102] Ermolenko SD, Khrapovitskii VG. Influence of air compressibility on the aerodynamic characteristics of a wing moving at subsonic speed near the Earth's Surface. Isvestiya VUZ Aviatsionnaya Tekhnika, No. 4; 1967.

[103] Thiery M, Coustols E. URANS computations of shock-induced oscillations over 2D rigid airfoils: influence of test section geometry. Flow Turbul Combust 2005;74(4):331-54.

[104] Doig G, Barber TJ, Neely AJ. Aerodynamic characteristics of a swept wing in close ground proximity at high subsonic mach numbers. J Aerosp Eng 2012;25:600-12.

[105] Cho J, Han C. Unsteady trailing vortex evolution behind a wing in ground effect. J Aircr 2005;42(2):429-34.

[106] Rozhdestvensky KV. Aerodynamics of a lifting system in extreme ground effect (text). 1st ed.. New York: Springer-Verlag; 2000.

[107] Post RF, Gurol S. The Inductrack approach to electromagnetic launching and Maglev trains. In: Sixth international symposium on magnetic levitation technology; 2001.

[108] Sharma T. Vacuum Maglev: an international endeavour for a global space program (Doctoral dissertation). University of Sussex; 2012.

[109] Mankins JC. The maglifter: an advanced concept using electromagnetic propulsion in reducing the cost of space launch. In: AIAA 30th Joint propulsion conference; 1994. p. 27-9.

[110] Yang W, Qiu M, Liu Y, Wen Z, Duan Y, Chen X. Levitation characteristics in an HTS maglev launch assist test vehicle. Superconduct. Sci. Technol. 2007;20 (3):281.

[111] Tomita N, Nebylov AV, Sokolov VV, Ohkami Y. Performance and technological feasibility of rocket powered HTHL-SSTO with take-off assist (aerospace plane/ekranoplane). Acta Astronaut. 1999;45(10):629-37.

[112] Menter FR. Two-equation eddy-viscosity turbulence models for engineering applications. AIAA J 1994:32(8):269-89.

[113] Doig G, Wang S, Young J, Kleine H. Aerodynamics of Transonic and Supersonic Projectiles in Ground Effect. In: 52nd AIAA aerospace sciences meeting, National Harbor, MD; 2014

[114] Doig G, Kleine H, Neely AJ, Barber TJ, Purdon JP, Appleby EM, et al. The aerodynamics of a supersonic projectile in ground effect. In: Proceedings of the 26th international symposium on shock waves, Göttingen, Germany; 2007.

[115] Weinstein LM, Minto D. Focusing schlieren photography at the Holloman high speed test track. In: 22nd International congress on high-speed photography and photonics; 1997. p. 865-873. 
[116] Lofthouse AJ, Hughson MC, Palazotto AN. Computational aerodynamic analysis of the flow field about a hypervelocity test sled; 2002.

[117] Praharaj SC, Roger RP. Aerodynamic computations of integrated missile-onsled vehicles. In: Proceedings of the 34th AIAA aerospace sciences meeting and exhibit, Reno, NV; 1996.

[118] Feltz LV, Rigali DJ. High-speed monorail rocket sleds for aerodynamic testing at high Reynolds numbers. J Spacecr Rocket 1968;5(11):1341-6.

[119] Ratliff, CL. Static force and moment test of the Holloman narrow-gage rocket sled at Mach numbers from 3.5 to $5.5 ; 1987$.
[120] Meinke R, Guiterrez H, Kirk D. Electromagnetic launching for affordable agile access to space. Melbourne, FL: Advanced Magnet Laboratory Inc; 2006.

[121] Intrieri PF, Malcolm GN. Ballistic range investigation of sonic-boom overpressures in water. AIAA J 1973;11(4):510-6.

[122] Doig G, Barber TJ, Leonardi E, Neely AJ, Kleine H. Aerodynamics of a supersonic projectile in proximity to a solid surface. AIAA J 2011;48(12):2916-30.

[123] Knight D, Yan H, Panaras AG, Zheltovodov A. Advances in CFD prediction of shock wave turbulent boundary layer interactions. Prog Aerosp Sci 2003;39 (2):121-84. 